\title{
Effect of Blood Flow Restriction on Functional, Physiological and Structural Variables of Muscle in Patients with Chronic Pathologies: A Systematic Review
}

\author{
Álvaro Jesús Reina-Ruiz ${ }^{1}$ (D) Alejandro Galán-Mercant ${ }^{2,3} * \mathbb{D}$, Guadalupe Molina-Torres ${ }^{4, *(\mathbb{D})}$,

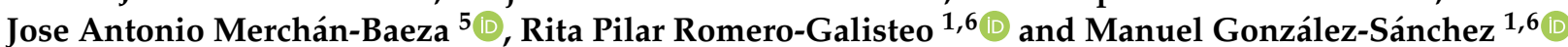

check for

updates

Citation: Reina-Ruiz, Á.J.;

Galán-Mercant, A.; Molina-Torres, G.;

Merchán-Baeza, J.A.;

Romero-Galisteo, R.P.;

González-Sánchez, M. Effect of Blood

Flow Restriction on Functional,

Physiological and Structural

Variables of Muscle in Patients with Chronic Pathologies: A Systematic Review. Int. J. Environ. Res. Public Health 2022, 19, 1160. https:// doi.org/10.3390/ijerph19031160

Academic Editors: Luis Javier Chirosa Ríos,

Daniel Jerez-Mayorga and

Pedro Delgado-Floody

Received: 1 December 2021

Accepted: 13 January 2022

Published: 20 January 2022

Publisher's Note: MDPI stays neutral with regard to jurisdictional claims in published maps and institutional affiliations.

Copyright: (C) 2022 by the authors. Licensee MDPI, Basel, Switzerland. This article is an open access article distributed under the terms and conditions of the Creative Commons Attribution (CC BY) license (https:// creativecommons.org/licenses/by/ $4.0 /)$.
1 Department of Physiotherapy, Faculty of Health Sciences, University of Málaga, Arquitecto Francisco Peñalosa, 3, 29071 Málaga, Spain; alvaro.reina.ruiz@gmail.com (Á.J.R.-R.); rpromero@uma.es (R.P.R.-G.); mgsa23@uma.es (M.G.-S.)

2 MOVE-IT Research Group, Department of Physical Education, Faculty of Education, Sciences University of Cádiz, 11002 Cádiz, Spain

3 Biomedical Research and Innovation Institute of Cádiz (INiBICA) Research Unit, Puerta del Mar University Hospital, University of Cádiz, 11002 Cádiz, Spain

4 Department of Nursing Science, Physiotherapy and Medicine, Faculty of Nursing and Physiotherapy, University of Almeria, 04120 Almeria, Spain

5 Centre for Health and Social Care Research (CESS), Research Group on Methodology, Methods, Models and Outcomes of Health and Social Sciences (M3O), Faculty of Health Science and Welfare, University of Vic-Central University of Catalonia (UVIC-UCC), C. Sagrada Família, 7, 08500 Vic, Spain; josan.merchan@uvic.cat

6 Instituto de Investigación Biomédica de Málaga, IBIMA, Calle Doctor Miguel Díaz Recio, 28, 29010 Málaga, Spain

* Correspondence: alejandro.galan@uca.es (A.G.-M.); gmt940@ual.es (G.M.-T.)

\begin{abstract}
The main objective of this systematic review of the current literature is to analyze the changes that blood flow restriction (BFR) causes in subjects with neuro-musculoskeletal and/or systemic pathologies focusing on the following variables: strength, physiological changes, structural changes and cardiocirculatory variables. The search was carried out in seven databases, including randomized clinical trials in which therapeutic exercise was combined with the blood flow restriction tool in populations with musculoskeletal pathologies. Outcome variables are strength, structural changes, physiological changes and cardiocirculatory variables. Twenty studies were included in the present study. Although there is a lot of heterogeneity between the interventions and evaluation instruments, we observed how the restriction of blood flow presents significant differences in the vast majority of the variables analyzed. In addition, we observed how BFR can become a supplement that provides benefits when performed with low intensity, similar to those obtained through highintensity muscular efforts. The application of the BFR technique can provide benefits in the short and medium term to increase strength, muscle thickness and cardiovascular endurance, even improving the physiological level of the cardiovascular system. In addition, BFR combined with low-load exercises also achieves benefits comparable to high-intensity exercises without the application of BFR, benefiting patients who are unable to lift high loads.
\end{abstract}

Keywords: blood flow restriction; musculoskeletal disease; controlled tourniquet; chronic pathologies; occlusion resistance training

\section{Introduction}

For decades, blood flow restriction (BFR), originating from Japan, has become one of the most popular techniques in physiotherapy [1]. To perform the BFR technique, a controlled tourniquet is performed, generating a gradual mechanical pressure just below it, affecting blood flow (both arterial and venous) and generating a hypoxia in the restricted 
area. For the realization of the tourniquet, mechanical cuffs are used, which are inflated and regulate the pressure generated in the compression zone, although nylon or elastic cuffs are also used for the realization of the tourniquet [2-5]. This zone of compression is usually the proximal region of both the upper and lower limbs. For the application of BFR, different levels of arterial occlusion pressure are used depending on the intended objectives. In this sense, two studies published in recent years have helped to determine the qualitative and quantitative aspects of the application of BFR, both in its application as part of training [4] and as part of a rehabilitation [3] protocol.

BFR is used in combination with strength exercise-low loads of around $20-40 \%$ of the maximum repetition that a subject can carry out are used-or through aerobic exercise at an intensity perceived by the subject as low-to-moderate [6]. Furthermore, BFR uses a series of cuffs that occlude the proximal parts of the extremities in order to cause restrictions in the arterial and venous blood flow, and through this phenomenon obtain the same benefits as high-load exercise [7]. The theories by which these results are supported are based on the metabolic effects induced during the hypoxic state that are created by the cuffs, neuromuscular and hormonal reactions [8]. The first theory is based on the increased production of lactate, reactive oxygen free radicals and nitrogen oxide. This in turn leads to greater protein synthesis and muscle recruitment and less protein destruction [9].

In the field of physiotherapy, BFR is becoming increasingly important since it has sparked interest from its application in training, in which it can be extrapolated to therapeutic exercise [10]. Among the methodologies used by this technique, there is heterogeneity in terms of the results as a consequence of the type of occlusion used, pressure of the arterial occlusion or pressure of the limb occlusion, the type of material used, the width of the cuff, the quantity of applied pressure (pressures between $40 \%$ and $80 \%$ are generally exerted) and the training method (aerobic or strength) [11,12]. For the application of BFR cuffs, it is acknowledged that the understanding of the comorbidities that the individual may present is important, emphasizing deep vein thrombosis as a contraindication, unless there is medical authorization; and rhabdomyolysis, which appears as a result of overexertion and is known to have a low incidence of $0.07-0.2 \%$ [4].

Within BFR, it is known that its application has led to the obtainment of greater gains in muscle mass and strength of subjects seeking to improve their performance such as recovering from an injury in its initial stages [13]. For this reason, BFR is considered an important tool for those patients experiencing problems during recovery, since they may present exacerbated symptoms with high-load exercises, or long-term immobilization that may lead to muscle atrophy, simultaneously fulfilling the goals of maintaining or increasing muscle mass and strength [13-15]. In addition, this working method can create improvements in the ability to contract muscles to a greater extent, although it has been proven in healthy adults who practice exercise [16] that its benefits depend entirely on the type of exercise performed [aerobic or strength] and the population practicing it [17]. On the other hand, it is reported that BFR can have short-term direct effects on aerobic capacity [18,19] and on the vascular system, presenting a hypotensive effect and increased heart rate and cardiovascular function [20,21].

The body of evidence regarding BFR has substantially increased over the last two decades, including the evaluation of the performance and, to a lesser extent, muscle hypertrophy in healthy subjects and focusing on the evaluation of other variables $[6,22]$. The most recent reviews collected information regarding physiological changes such as aerobic capacity and structural changes such as muscle cross-sectional area, which provide more information to the currently consolidated evidence $[23,24]$. However, as of yet no systematic review has analyzed the structural changes produced by BFR regarding strength, as well as metabolic and cardiovascular changes [14,25,26].

To our knowledge, no review exists that explains how BFR influences strength, physiological and structural changes, as well as cardiocirculatory variables in subjects with pathologies. In accordance with the aforementioned points, the main objective of this systematic review of the current literature will be to analyze the changes caused by BFR in subjects with 
neuro-musculoskeletal and/or systemic pathologies, focusing on the following variables: strength, physiological changes, structural changes and cardiocirculatory variables.

\section{Materials and Methods}

In this study, a systematic review based on current scientific evidence is carried out using PRISMA guideline.

\subsection{Search Strategy}

During the process of developing the search strategy for this systematic review, information collected from various databases was used: PubMed, Embase, Cochrane, PEDro, CINAHL, Scopus, SPORTDiscus and Trip Medical Database. The selected articles were filtered using the following keywords: kaatsu; ischemic training; blood flow restriction; occlusion resistance training; vascular occlusion; vascular restriction; chronic disease. Boolean indicators AND and OR were combined to perform the search.

\subsection{Document Selection}

Once the search strategy was carried out, the inclusion criteria of studies were established as those randomized clinical trials in which therapeutic exercise was combined with the blood flow restriction tool in populations with musculoskeletal pathologies, and which were published between 1 January 2015 and 21 August 2021.

All studies that had been published in a language other than Spanish, English, Italian, French and Portuguese were established as exclusion criteria. Furthermore, those studies whose score on the PEDro scale was less than 6 were excluded.

\subsubsection{Selection Method}

Scientific literature searches across the different databases and the selection of the above-mentioned documents-applying the inclusion and exclusion criteria-were carried out in parallel with two blinded researchers. In the event that there was any discrepancy between the authors, the selection of the document was resolved by a third blinded investigator.

\subsubsection{Methodological Quality}

In relation to the methodological quality assessment of the randomized clinical trials, the PEDro assessment scale was used, which consists of 11 questions, 10 of which (selection criteria; selection randomization: hidden allocation; initial comparability between groups; totality of blinded subjects; all therapists blinded; all evaluators blinded; adequacy of follow-up; analysis with intention to treat; comparison of results between groups; existence of specific measures and variability) can be answered in a dichotomous way using Yes/No, which is awarded depending on the fulfillment of the requirements of the particular point. Regarding the unscored question, this cannot be entered due to external validity influences which do not meet the internal validity requirement [27].

In addition, a distinction was made between studies of high (score greater than or equal to 6 points) and low (score lower than 6 points) methodological quality based on the results obtained from this scale [27].

\subsection{Result Variables}

The following were selected as outcome variables for the subsequent analysis of the studies: strength, structural changes, physiological changes and cardiocirculatory variables. The strength variable is defined as the ability of the muscles to produce a muscular contraction in the absence or against a load with an effort to counteract a force, and this can be evaluated in any of its modalities: isometric, isotonic or isokinetic contraction [28,29]. For structural changes, this variable is understood as the area perpendicular to the muscle fibers compared to the total area of the muscle. In addition, its presence, in a greater quantity, 
is considered a predictor of greater strength, which is why it has a direct relationship with strength [30,31].

The variable of physiological changes is defined as the amount of physical energy used during any physical activity by any type of person compared to rest [32]. In contrast, a cardiocirculatory variable is understood to be the measurable amount in a blood sample or imaging test of certain substances, cells or molecules present in the bloodstream to detect blood markers [33].

According to the analysis of the results of each study variable, a temporal continuity model was applied in order to typify the information collected and thus be able to homogenize the presentation of each data obtained. Subsequently, the values of the results were relativized to $100 \%$ on a scale of $0-100$ in order to allow comparison between the different results.

Within the continuity model, the so-called baseline was proposed as the moment of data collection just before the intervention group participants could begin. Subsequently, data collection was divided into four moments: short-term, medium-term, long-term and follow-up period. For the short term, the period between the start of treatment and week 6 is attributed; medium term: from week 6 to week 12; long term: between weeks 12 and 24; finally, for the follow-up period: a period of time after the applied intervention that spans more than 24 weeks.

\section{Results}

Figure 1 shows the results of the search. The initial search identified 547 documents; after eliminating duplicate documents, 367 documents were analyzed for an initial evaluation. After the initial evaluation and after a complete reading of the document and analysis of the outcome variables, 20 documents were selected and included in this study. For more details on the document-filtering process, see Figure 1.

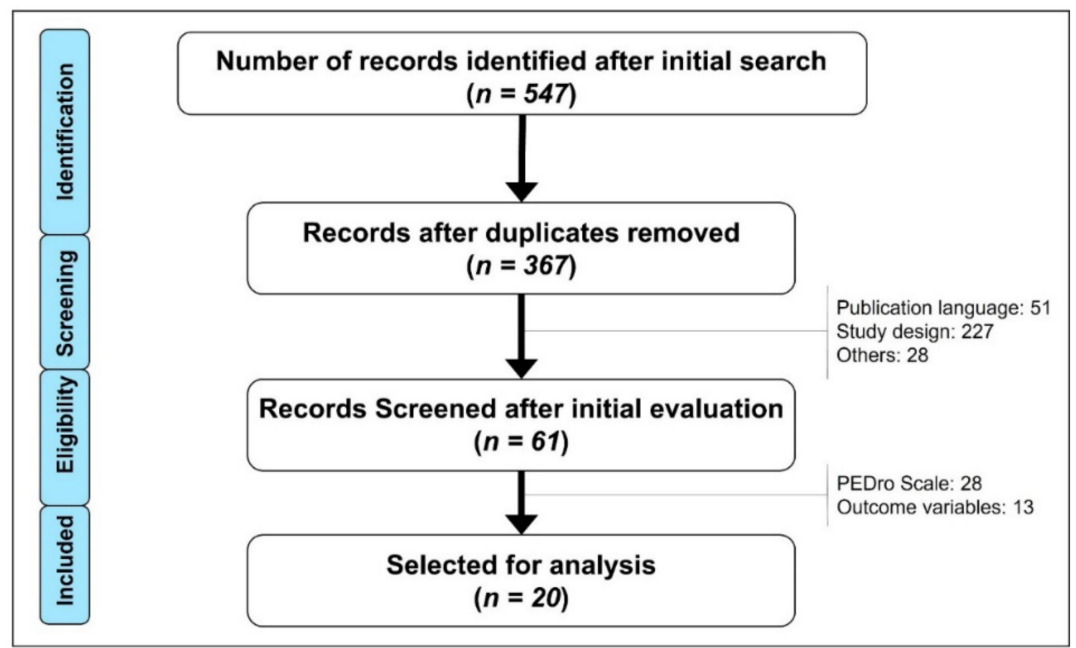

Figure 1. Flow chart of the search, filtering and selection of the included documents.

Table 1 presents a minimum sample size of 20 patients [34] and a maximum of 79 [35], in which the mean age of the participants was 45 years, ranging from 14 [36] to 76 years [37]. Likewise, when the blood flow restriction cuff was placed, a minimum pressure of $30 \%$ of the total blood flow restriction was applied [38] and a maximum of $80 \%$ [36]. The interventions used were strength, aerobic type and blood flow restriction training. Regarding the frequency of the sessions, the minimum follow-up was 4 weeks $[39,40]$ and the longest was 16 weeks [35]. Furthermore, the minimum recurrence was two weekly sessions [41] and the maximum was three weekly sessions [42]. The variables evaluated were strength, structural and physiological changes of the muscle and cardiovascular variables at the blood level. 
Table 1. Study characteristics.

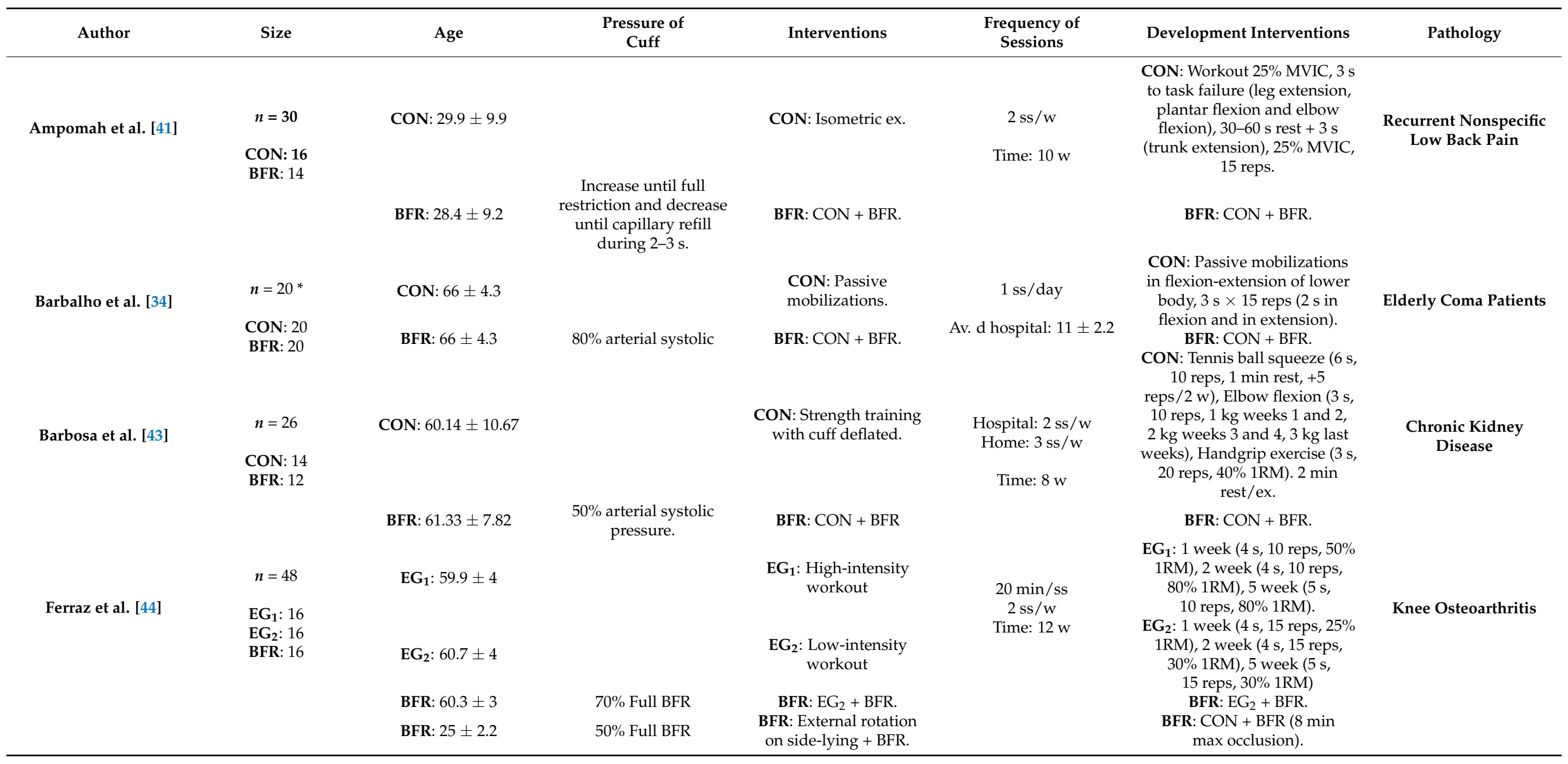


Table 1. Cont.

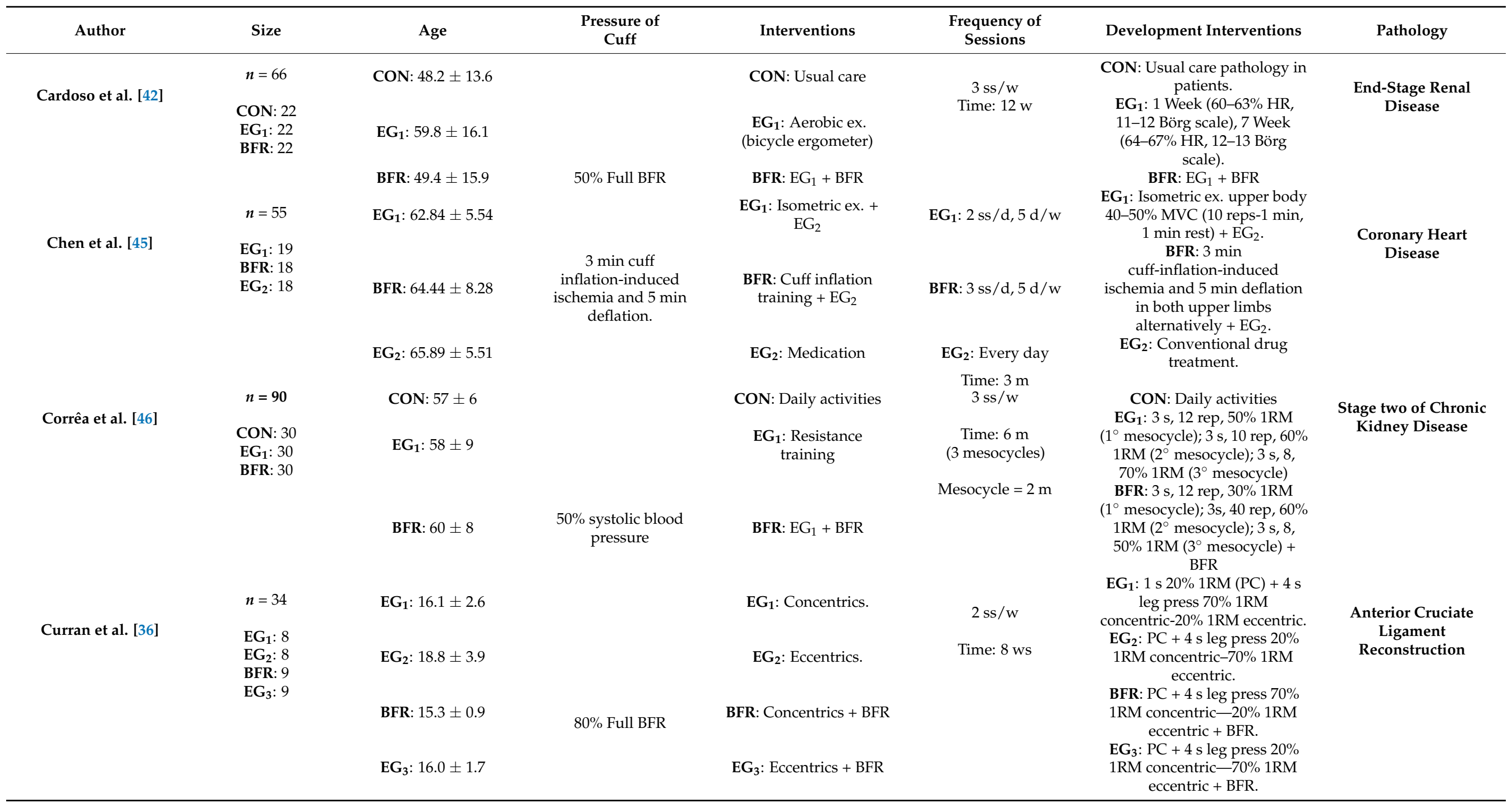


Table 1. Cont.

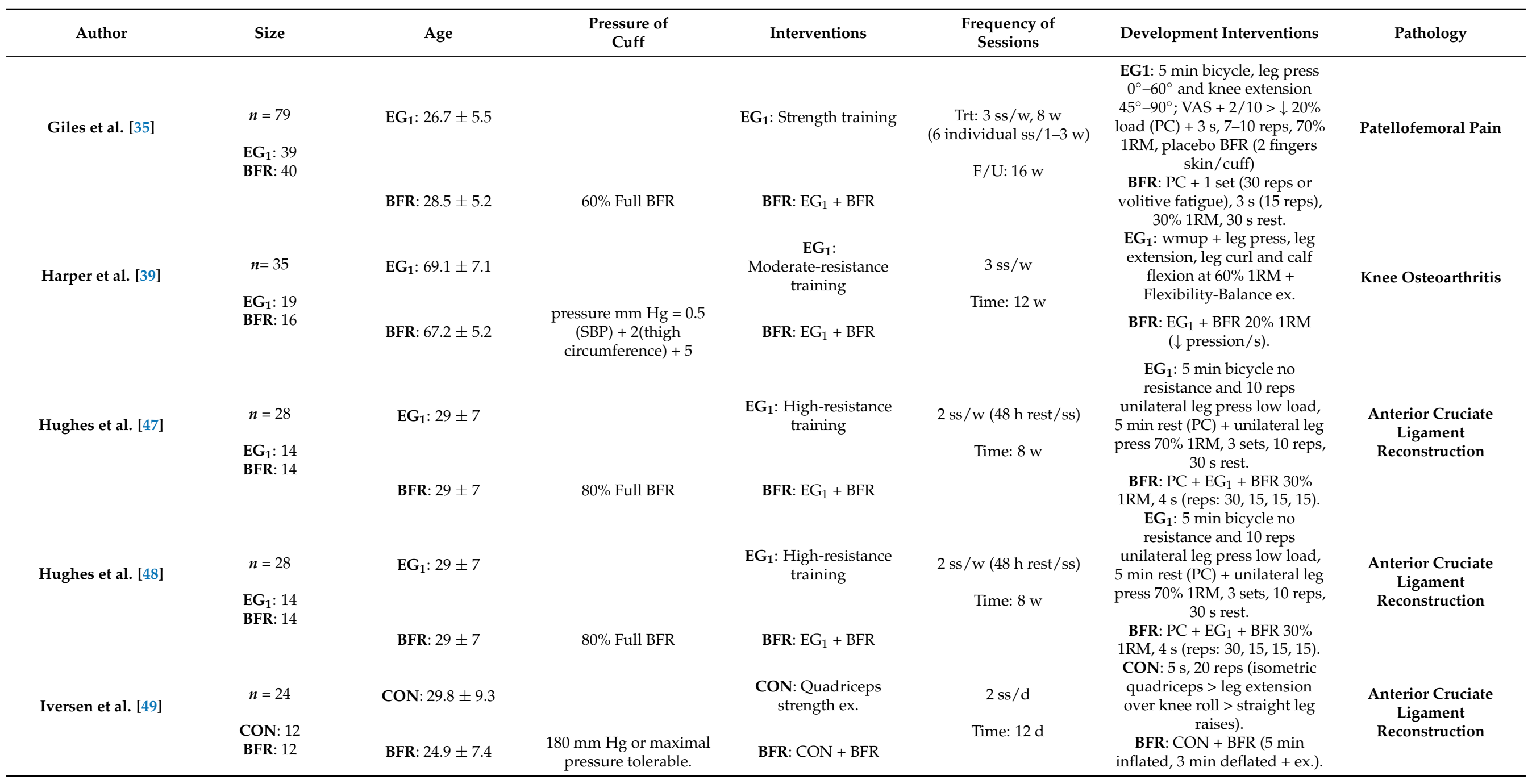


Table 1. Cont.

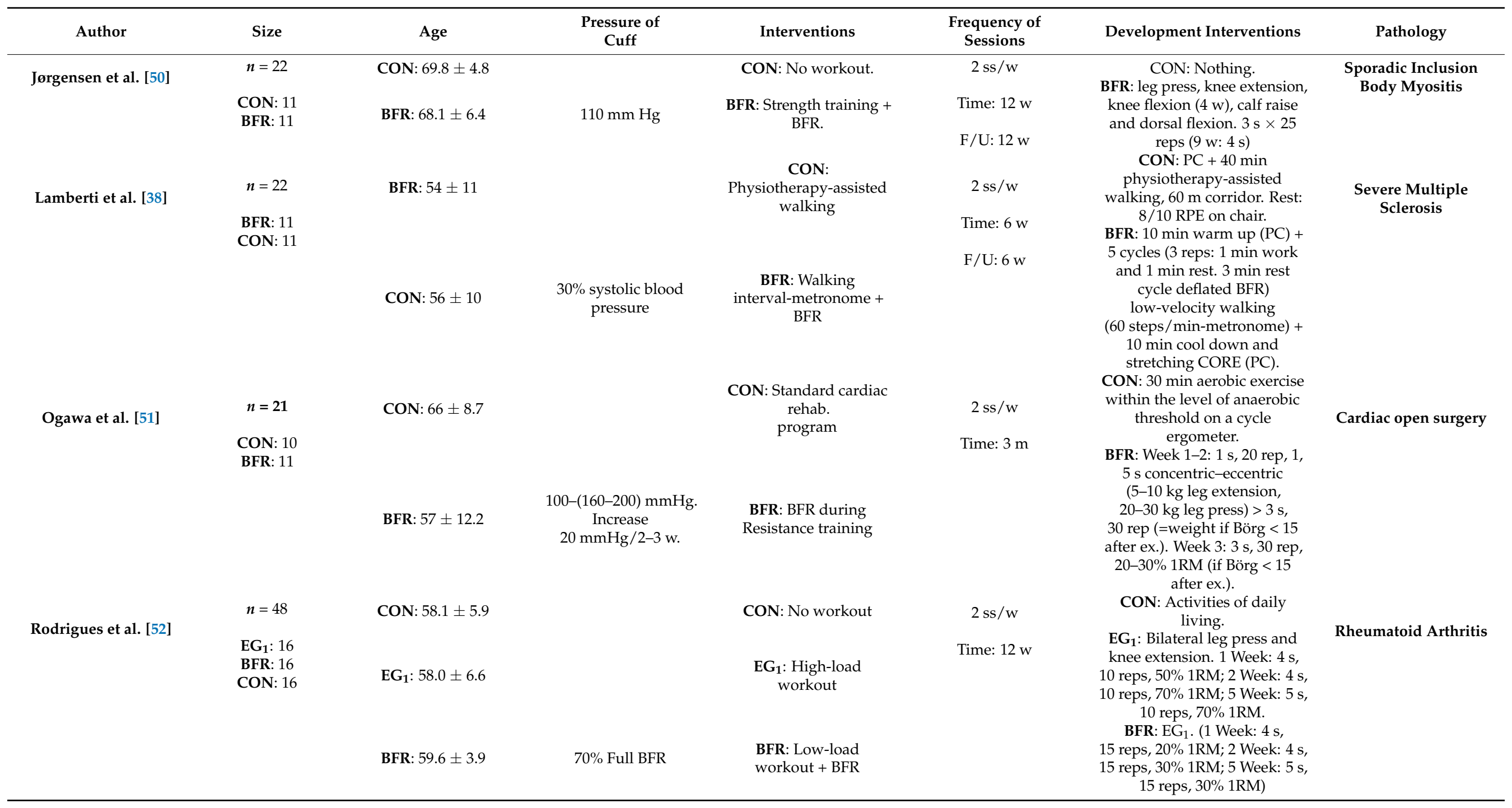


Table 1. Cont.

\begin{tabular}{|c|c|c|c|c|c|c|c|}
\hline Author & Size & Age & $\begin{array}{l}\text { Pressure of } \\
\text { Cuff }\end{array}$ & Interventions & $\begin{array}{l}\text { Frequency of } \\
\text { Sessions }\end{array}$ & Development Interventions & Pathology \\
\hline \multirow[t]{2}{*}{ Segal et al. [39] } & $n=42$ & CON: $56.1 \pm 7.7$ & & $\begin{array}{l}\text { CON: Low-load } \\
\text { workout }\end{array}$ & $3 \mathrm{ss} / \mathrm{w}$ & $\begin{array}{l}\text { CON: Leg press } 30 \% 1 \mathrm{RM}: 4 \mathrm{~s} \\
\text { (reps: } 30,15,15,15), 30 \mathrm{~s} \text { rest. } \\
\text { Rep: } 2 \mathrm{~s} \text { concentric and } 2 \mathrm{~s}\end{array}$ & Knee Osteoarthritis \\
\hline & $\begin{array}{l}\text { CON: } 22 \\
\text { BFR: } 20\end{array}$ & BFR: $58.4 \pm 8.7$ & $\begin{array}{l}1 \text { Week: } 160 \mathrm{~mm} \mathrm{Hg} \\
2 \text { Week: } 180 \mathrm{~mm} \mathrm{Hg} \\
3 \text { Week: } 200 \mathrm{~mm} \mathrm{Hg}\end{array}$ & BFR: $C O N+B F R$. & $\begin{array}{l}\text { Time: } 4 \mathrm{w} \\
\text { F/U: } 3 \mathrm{~d}\end{array}$ & $\begin{array}{c}\text { eccentric. } \\
\text { BFR: CON + BFR. }\end{array}$ & \\
\hline \multirow[t]{2}{*}{ Segal et al. [40] } & $n=45$ & CON: $54.6 \pm 6.9$ & & $\begin{array}{l}\text { CON: Low-load } \\
\text { workout }\end{array}$ & $\begin{array}{l}3 \mathrm{ss} / \mathrm{w} \\
\text { Time: } 4 \mathrm{w}\end{array}$ & $\begin{array}{c}\text { CON: Leg press } 30 \% 1 \mathrm{RM}: 4 \mathrm{~s} \\
\text { (reps: } 30,15,15,15), 30 \mathrm{~s} \text { rest. } \\
\text { Rep: } 2 \text { s concentric, } 2 \mathrm{~s} \\
\text { eccentric. }\end{array}$ & Knee Osteoarthritis \\
\hline & BFR: 21 & BFR: $56.1 \pm 5.9$ & $\begin{array}{l}1 \text { Week: } 160 \mathrm{~mm} \mathrm{Hg} \\
2 \text { Week: } 180 \mathrm{~mm} \mathrm{Hg} \\
3 \text { Week: } 200 \mathrm{~mm} \mathrm{Hg}\end{array}$ & BFR: $C O N+B F R$. & $\mathrm{F} / \mathrm{U}: 3 \mathrm{~d}$ & BFR: $\mathrm{CON}+\mathrm{BFR}$. & \\
\hline \multirow[t]{2}{*}{ Tennent et al. [53] } & $n=24$ & CON: $37.0(32-47)$ & & CON: Physiotherapy & & $\begin{array}{l}\text { CON: Immediate weight } \\
\text { loading, immediate formal } \\
\text { physiotherapy and no range }\end{array}$ & $\begin{array}{l}\text { Non-Reconstructive } \\
\text { Knee Arthroscopy }\end{array}$ \\
\hline & $\begin{array}{l}\text { CON: } 13 \\
\text { BFR: } 11\end{array}$ & BFR: $37.0(30-46.2)$ & $80 \%$ Full BFR & $\begin{array}{c}\text { BFR: Physiotherapy + } \\
\text { (Strength training + } \\
\text { BFR) }\end{array}$ & Time: 6 ss & $\begin{array}{l}\text { of motion restrictions. } \\
\text { BFR: CON + } 4 \text { sets (reps: } 30, \\
\text { 15, } 15,15), 30 \% 1 R M, 30 \mathrm{~s} \\
\text { rest-1 min rest/ex. (leg press, } \\
\text { leg extension and reverse } \\
\text { press). } 5 \text { min max. } \\
\text { occlusion/ex. }\end{array}$ & \\
\hline
\end{tabular}

BFR: Blood flow restriction group; CON: Control Group; d: days; EG 1 : Experimental group 1; EG 2 Experimental group 2; EG 3 : Experimental group 3; h: hours; HR: Heart Rate kg: Kilogram; PC: Common process; MVIC: Maximal voluntary isometric contraction; reps: Repetitions; RM: Maximal repetition; RPE: Rating Perceived Exertion; s: Seconds; ss: SessionsVAS: Visual Analogic Scale; w: Weeks. * The author classifies subjects depending on their lower limbs. 
The results of the strength variable are shown in Table 2, using a total of six assessment tools such as the dynamometer [42,44,45], 1 maximum repetition [46,47], 10 maximum repetitions [46], central activation ratio [37], manual muscle test [48] and kinetic communicator [48]. Among all, the dynamometer is the most used, presenting significant heterogeneity as a consequence of the use of different units of measurements and assessment of different anatomical areas, together with the estimation of 1 maximum repetition, where its mean is $112.83 \mathrm{~kg}$, varying between $0.66 \mathrm{~kg}$ [37] and $430.6 \mathrm{~kg}$ [41]; however, it does not present homogeneity in the evaluation units or the results, due to the variety of exercises used. In addition, all the studies present an intervention duration of up to 6 months and a follow-up of 3 months in a total of five studies.

When it comes to analyzing structural changes in muscle (Table 3), a high heterogeneity is observed in the assessment instruments and, therefore, in the outcome variables. In this sense, imaging techniques are used preferentially, although the outcome variable obtained sometimes allows a comparison between the different studies that use the same technique, but only an approximation to the eventual comparison of the results between the different techniques. Four studies used magnetic resonance imaging, two used computerized tomography and five used ultrasound. However, in the latter instrument, three studies analyzed cross-sectional area while three analyzed muscle thickness (one study, Barbalho et al. [35], analyzed both outcome variables). All imaging techniques are widely used in diagnosis and clinical follow-up; however, at the same time, they all present a series of strengths and weaknesses that must be taken into account when interpreting the results. Table 3 shows structural muscle changes; data were collected from the following four assessment tools: intranuclear magnetic resonance [36,49], ultrasound [37], measuring tape $[35,44,50]$ and computerized tomography $[46,47]$. Of the different assessment tools, both intranuclear magnetic resonance imaging and ultrasound showed significant heterogeneity between their results as a consequence of using different units of measurement in the data-collection step, as well as the different muscle groups assessed. Computerized tomography presented an average of $4787 \mathrm{~mm}^{2}$, with minimum and maximum values of 3825 and $5750 \mathrm{~mm}^{2}$, respectively [46], therefore presenting homogeneity in its results, although they were shown in only two studies. Meanwhile, in the measuring tape an average value of $43.84 \mathrm{~cm}$ was collected, with values between 23.67 [44] and $64 \mathrm{~cm}$, with minor homogeneity due to assessment in different parts of the body or target populations [50]. In general, all studies collected data during the intervention up to the third month and subsequently did not collect data at the follow-up, except for the studies by Ampomah et al. and Segal et al. which carried out data collection in the first three months.

Table 4 shows data regarding the variable physiological changes of the muscle, in which up to four assessment tools were used: the Fatigue Severity Scale [39], Modified Fatigue Impact Scale [39], Rating Perceived Exertion Scale [51] and accelerometer [47]. For the subvariable fatigue, a disparity was observed between the results, since two scales and a different range of assessment were used in the same study. As for the other subvariables, a direct comparison cannot be made since there are insufficient studies to evaluate it or the results were not collected at some points of the intervention. However, the studies collected results during the first 3 months of intervention and there is only one study that employed a follow-up of a duration equal to 3 months.

For the cardiocirculatory variables shown in Table 5, different measuring instruments were used, such as ultrasound [44], cardiopulmonary exercise test [45], electrocardiogram [52] and electronic manometer [52,53]. For any of the tools observed in Table 5, an average assessment of the results obtained in each of the subvariables cannot be made since, for the most part, all the data were collected in a single study, so it is not possible to directly evaluate. Both studies collected data from the start of the intervention to 3 months, without assessing the post-intervention results by follow-up. 
Table 2. Strength.

\begin{tabular}{|c|c|c|c|c|c|c|c|c|c|}
\hline \multirow{2}{*}{$\begin{array}{l}\text { Measurement } \\
\text { Tool }\end{array}$} & \multirow{2}{*}{ Article } & \multirow{2}{*}{ Group } & \multirow{2}{*}{ Baseline } & \multicolumn{3}{|c|}{ Measurements (SD/CI 95\%) } & \multicolumn{3}{|c|}{ Follow-Up (SD/CI 95\%) } \\
\hline & & & & 0-6 Weeks & 6-12 Weeks & 3-6 Months & 1-3 Months & 3-6 Months & $>6$ Months \\
\hline \multirow{31}{*}{ Dynamometer } & \multirow{2}{*}{$\begin{array}{l}\text { Ampomah et al. } \\
{[41](\mathrm{Nm})}\end{array}$} & $\mathrm{CON}$ & $\begin{array}{c}\text { TE: } 238.2 \pm 97.9 \\
\text { LE: } 939.9 \pm 301.0\end{array}$ & - & $8.4 \% \pm 8.2 \% \wedge$ & - & $14.3 \% \pm 6.2 \%^{\wedge}$ & - & - \\
\hline & & BFR & $\begin{array}{c}\text { TE: } 221.2 \pm 86.1 \\
\text { LE: } 904.1 \pm 277.5\end{array}$ & - & $4.7 \% \pm 7.9 \%$ & - & $-0.1 \% \pm 5.5 \%^{\wedge}$ & - & \\
\hline & \multirow{2}{*}{$\begin{array}{c}\text { Barbosa et al. [43] } \\
\text { (kgf) }\end{array}$} & $\mathrm{CON}$ & $24.93(19.76-30.10)$ & - & $27.29(22.11-32.46)$ & - & - & - & - \\
\hline & & BFR & $26.83(21.18-32.48)$ & - & $29.08(23.86-34.30)$ & - & - & - & - \\
\hline & \multirow{2}{*}{$\begin{array}{l}\text { Cardoso et al. [42] } \\
\text { (kgf) }\end{array}$} & $\mathrm{CON}$ & $53.8 \pm 26.6$ & - & $53.9 \pm 35.7$ & - & - & - & - \\
\hline & & $\mathrm{EG}_{1}$ & $70.97 \pm 27.12$ & - & $70.28 \pm 27.57$ & - & - & - & - \\
\hline & \multirow{2}{*}{$\begin{array}{l}\text { Giles et al. [35] } \\
\qquad(\mathrm{Nm})\end{array}$} & $\mathrm{EG}_{1}$ & $135.1 \pm 55.1$ & - & $158.7 \pm 57.4$ & - & - & - & - \\
\hline & & BFR & $131.2 \pm 61.9$ & - & $166.4 \pm 59.4$ & - & - & - & - \\
\hline & \multirow{6}{*}{$\begin{array}{l}\text { Harper et al. [37] } \\
\text { (Nm) }\end{array}$} & $\mathrm{EG}_{1}$ & $44.2 *$ & $52.5 \pm 3^{*}$ & $54.75 \pm 2.5^{*}$ & - & - & - & - \\
\hline & & BFR & $44.5 *$ & $50.5 \pm 3 *$ & $53 \pm 3.5 *$ & - & - & - & - \\
\hline & & & $60^{\circ} / \mathrm{seg}$ & & $60^{\circ} / \mathrm{seg}$ & & & & \\
\hline & & & I: $1.8 \pm 0.5^{*}$ & & I: $1.35 \pm 0.5^{*}$ & & & & \\
\hline & & & NI: $2.05 \pm 0.2 *$ & & NI: $2.35 \pm 0.3 *$ & & & & \\
\hline & & & $150^{\circ} / \mathrm{seg}$ & & $150^{\circ} / \mathrm{seg}$ & & & & \\
\hline & \multirow{13}{*}{$\begin{array}{l}\text { Hughes et al. [47] } \\
\text { (N.kg bm) }\end{array}$} & $\mathrm{EG}_{1}$ & I: $1.47 \pm 0.3^{*}$ & - & I: $1.25 \pm 0.25^{*}$ & - & - & - & - \\
\hline & & & NI: $1.65 \pm 0.3 *$ & & NI: $1.87 \pm 0.35^{*}$ & & & & \\
\hline & & & $300^{\circ} / \mathrm{seg}$ & & $300^{\circ} / \mathrm{seg}$ & & & & \\
\hline & & & NI: $1.65 \pm 0.25 *$ & & NI: $1.85 \pm 0.35$ * & & & & \\
\hline & & & $60^{\circ} / \mathrm{seg}$ & & $60^{\circ} / \mathrm{seg}$ & & & & \\
\hline & & & I: $1.75 \pm 0.45^{*}$ & & I: $1.6 \pm 0.5^{*}$ & & & & \\
\hline & & & NI: $2.25 \pm 0.3 *$ & & NI: $2.45 \pm 0.2 *$ & & & & \\
\hline & & & $150^{\circ} / \mathrm{seg}$ & & $150^{\circ} / \mathrm{seg}$ & & & & \\
\hline & & BFR & I: $1.37 \pm 0.28^{*}$ & - & I: $1.42 \pm 0.28^{*}$ & - & - & - & - \\
\hline & & & NI: $1.65 \pm 0.2 *$ & & NI: $1.8 \pm 0.25^{*}$ & & & & \\
\hline & & & $300^{\circ} / \mathrm{seg}$ & & $300^{\circ} / \mathrm{seg}$ & & & & \\
\hline & & & I: $1.05 \pm 0.2 *$ & & I: $1.1 \pm 0.17^{*}$ & & & & \\
\hline & & & NI: $1.65 \pm 0.22 *$ & & NI: $1.83 \pm 0.28 *$ & & & & \\
\hline & Segal et al. (2015) & BFR & $169.7 \pm 39.0$ & $-0.1 \pm 3.3$ & - & - & $0.4 \pm 2.4 \cdots, \wedge$ & - & - \\
\hline & [39] (Nm) & $\mathrm{CON}$ & $151.9 \pm 34.8$ & $7.0 \pm 3.0 *$ & - & - & $6.7 \pm 2.3 \cdots, \wedge$ & - & - \\
\hline & Segal et al. (2015) & BFR & $1.3 \pm 0.3$ & - & - & - & $0.07 \pm 0.03$ & - & - \\
\hline & [40] (Nm) & $\mathrm{CON}$ & $1.3 \pm 0.4$ & - & - & - & $0.05 \pm 0.03$ & - & - \\
\hline
\end{tabular}


Table 2. Cont.

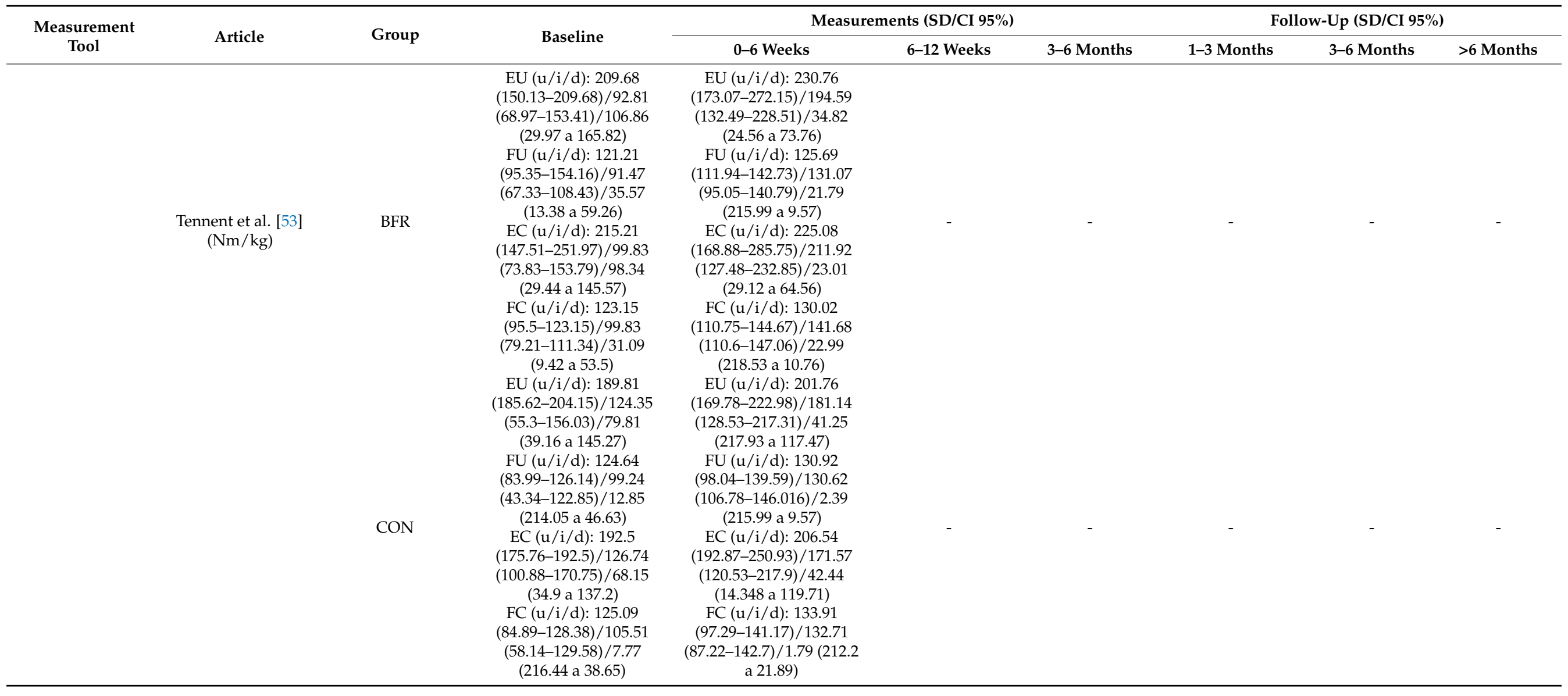


Table 2. Cont.

\begin{tabular}{|c|c|c|c|c|c|c|c|c|c|}
\hline \multirow{2}{*}{$\begin{array}{c}\text { Measurement } \\
\text { Tool }\end{array}$} & \multirow{2}{*}{ Article } & \multirow{2}{*}{ Group } & \multirow{2}{*}{ Baseline } & \multicolumn{3}{|c|}{ Measurements (SD/CI 95\%) } & \multicolumn{3}{|c|}{ Follow-Up (SD/CI 95\%) } \\
\hline & & & & 0-6 Weeks & 6-12 Weeks & 3-6 Months & 1-3 Months & 3-6 Months & $>6$ Months \\
\hline & \multirow{4}{*}{$\begin{array}{l}\text { Curran et al. [36] } \\
\qquad(\mathrm{Nm})\end{array}$} & $\mathrm{EG}_{1}$ & - & - & $\begin{array}{l}\text { ik: }-19.2 \pm 35.9 \\
\text { is: }-13.7 \pm 42.6\end{array}$ & - & - & - & - \\
\hline & & $\mathrm{EG}_{2}$ & - & - & $\begin{array}{l}\text { ik: }-10.8 \pm 34.7 \\
\text { is: }-10.0 \pm 36.3\end{array}$ & - & - & - & - \\
\hline & & BFR & - & - & $\begin{array}{l}\text { ik: }-16.7 \pm 21.4 \\
\text { is: }-18.0 \pm 34.5\end{array}$ & - & - & - & - \\
\hline & & $\mathrm{EG}_{3}$ & - & - & $\begin{array}{l}\text { ik: }-8.1 \pm 16.9 \\
\text { is: }-14.6 \pm 29.3\end{array}$ & - & - & - & - \\
\hline & \multirow{2}{*}{$\begin{array}{l}\text { Corrêa et al. [46] } \\
\text { (kgf) }\end{array}$} & $\mathrm{EG}_{1}$ & $22.2 \pm 5.8$ & - & - & $29.1 \pm 3.7$ & - & - & - \\
\hline & & BFR & $24.1 \pm 4.9$ & - & - & $30.2 \pm 3.2$ & - & - & - \\
\hline & \multirow{3}{*}{$\begin{array}{c}\text { Ogawa et al. [51] } \\
\text { (kgf) }\end{array}$} & $\mathrm{CON}$ & $\begin{array}{l}\text { HG: } 31.3(7.4) \\
\text { KE: } 33.5(10.5)\end{array}$ & $\begin{array}{l}\text { HG: } 28.3(8.2) \\
\text { KE: } 28(10.4)\end{array}$ & $\begin{array}{l}\text { HG: } 30.7(6.7) \\
\text { KE: } 31.7(7.48)\end{array}$ & - & - & - & - \\
\hline & & \multirow{2}{*}{ BFR } & HG: $30.3(7.5)$ & HG: $29.2(5.2)$ & HG: $33.9(8.5)$ & - & - & - & - \\
\hline & & & KE: 30.5 (11.2) & KE: $29.2(5.2)$ & KE: 41.8 (15.1) & - & - & - & - \\
\hline & \multirow{4}{*}{ Ferraz et al. [44] } & $\mathrm{EG}_{1}$ & $\begin{array}{l}\text { KE: } 33 \pm 9^{*} \\
\text { LP: } 130 \pm 45^{*}\end{array}$ & - & $\begin{array}{l}\text { KE: } 42.5 \pm 9^{*} \\
\text { LP: } 175 \pm 50 *\end{array}$ & - & - & - & - \\
\hline & & & KE: $31.5 \pm 11 *$ & & KE: $34 \pm 10 *$ & & & & \\
\hline & & $\mathrm{EG}_{2}$ & LP: $125 \pm 40^{*}$ & - & LP: $140 \pm 45^{*}$ & - & - & - & - \\
\hline & & BFR & $\begin{array}{l}\text { KE: } 33 \pm 8.5^{*} \\
\text { LP: } 125 \pm 30^{*}\end{array}$ & - & $\begin{array}{l}\text { KE: } 41 \pm 8^{*} \\
\text { LP: } 165 \pm 25^{*}\end{array}$ & - & - & - & - \\
\hline & \multirow{2}{*}{$\begin{array}{l}\text { Rodrigues et al. } \\
\qquad 52]\end{array}$} & $\mathrm{EG}_{1}$ & $\begin{array}{l}\text { KE: } 35 \pm 12.5^{*} \\
\text { LP: } 113 \pm 49.5^{*}\end{array}$ & - & $\begin{array}{l}\text { KE: } 43 \pm 12 * \\
\text { LP: } 140 \pm 45^{*}\end{array}$ & - & - & - & - \\
\hline & & BFR & $\begin{array}{l}\text { KE: } 30 \pm 12 * \\
\text { LP: } 110 \pm 30 *\end{array}$ & - & $\begin{array}{c}\text { KE: } 37.5 \pm 10.5^{*} \\
\text { LP: } 135 \pm 28^{*}\end{array}$ & - & - & - & - \\
\hline \multirow[t]{8}{*}{ 1RM (kg) } & \multirow{4}{*}{$\begin{array}{c}\text { Segal et al. (2015) } \\
\text { [39] }\end{array}$} & $\mathrm{CON}$ & $\begin{array}{l}\text { KE: } 33.5 \pm 12.5^{*} \\
\text { LP: } 112 \pm 35.5^{*}\end{array}$ & - & $\begin{array}{l}\text { KE: } 33.75 \pm 12 * \\
\text { LP: } 110 \pm 35^{*}\end{array}$ & - & - & - & - \\
\hline & & BFR & $346.1 \pm 95.5^{\mathrm{a}}$ & $11.3 \pm 14.0 *$ & - & - & $3.1 \pm 0.9{ }^{\cdots, \wedge}$ & - & - \\
\hline & & $\mathrm{CON}$ & $289.0 \pm 48.1^{\mathrm{a}}$ & $13.5 \pm 6.8^{*}$ & - & - & $4.7 \pm 1.3 \cdots, \wedge$ & - & - \\
\hline & & & $1 \mathrm{RM}(\mathrm{kg} / \mathrm{kg} \mathrm{bm}):$ & & & & $1 \mathrm{RM}(\mathrm{kg} / \mathrm{kg} \mathrm{bm}):$ & & \\
\hline & \multirow{4}{*}{$\begin{array}{c}\text { Segal et al. (2015) } \\
\text { [40] }\end{array}$} & BFR & $\begin{array}{c}2.3 \pm 0.6 \\
40 \% \text {. }\end{array}$ & 1RM $(\mathrm{kg} / \mathrm{kg} \mathrm{bm}):-$ & - & - & $0.4 \pm 0.3$ & - & - \\
\hline & & & $\begin{array}{c}40 \% 1 \mathrm{RM}(\mathrm{W} / \mathrm{kg} \mathrm{bm}): \\
12.6 \pm 2.5\end{array}$ & 40\% 1RM (W/ kg bm): - & & & $\begin{array}{l}40 \% \text { 1RM (W/kg } \\
\mathrm{bm}): 0.62 \pm 0.27\end{array}$ & & \\
\hline & & & $1 \mathrm{RM}(\mathrm{kg} / \mathrm{kg} \mathrm{bm}):$ & & & & $1 \mathrm{RM}(\mathrm{kg} / \mathrm{kg} \mathrm{bm}):$ & & \\
\hline & & $\mathrm{CON}$ & $\begin{array}{c}2.1 \pm 0.5 \\
40 \% 1 \mathrm{RM}(\mathrm{W} / \mathrm{kg} \mathrm{bm}): \\
11.3 \pm 2.9\end{array}$ & $\begin{array}{c}\text { 1RM (kg/kg bm): - } \\
40 \% 1 \mathrm{RM}(\mathrm{W} / \mathrm{kg} \mathrm{bm}):-\end{array}$ & - & - & $\begin{array}{c}0.2 \pm 0.3{ }^{*} \\
40 \% 1 \mathrm{RM}(\mathrm{W} / \mathrm{kg} \\
\mathrm{bm}): 0.42 \pm 0.26\end{array}$ & - & - \\
\hline
\end{tabular}


Table 2. Cont.

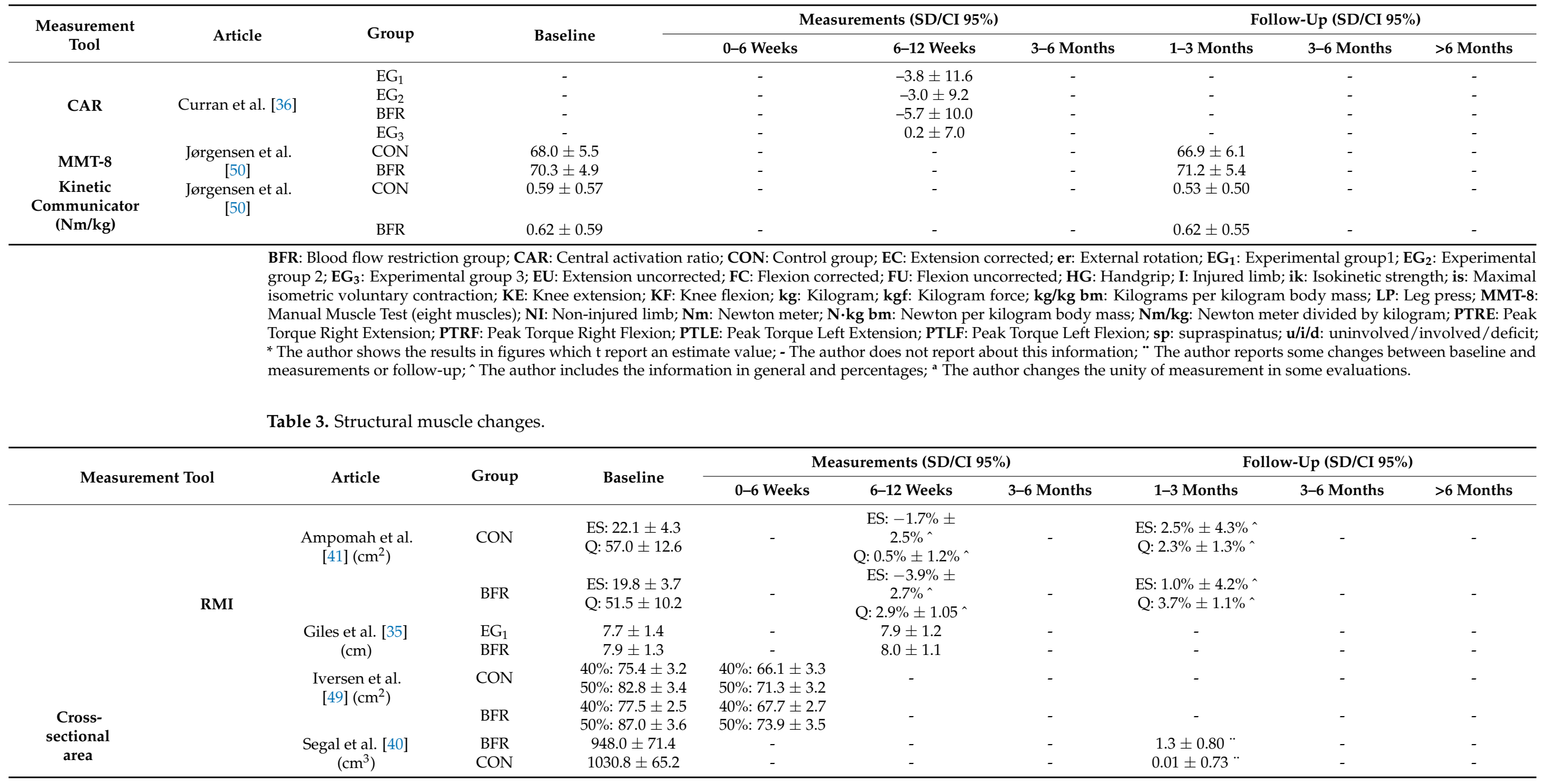


Table 3. Cont.

\begin{tabular}{|c|c|c|c|c|c|c|c|c|c|c|}
\hline \multirow{2}{*}{\multicolumn{2}{|c|}{ Measurement Tool }} & \multirow{2}{*}{ Article } & \multirow{2}{*}{ Group } & \multirow{2}{*}{ Baseline } & \multicolumn{3}{|c|}{ Measurements (SD/CI 95\%) } & \multicolumn{3}{|c|}{ Follow-Up (SD/CI 95\%) } \\
\hline & & & & & 0-6 Weeks & 6-12 Weeks & 3-6 Months & 1-3 Months & 3-6 Months & $>6$ Months \\
\hline \multirow{5}{*}{\multicolumn{2}{|c|}{ Ultrasound }} & Barbalho et al. & $\mathrm{CON}$ & $11.2 \pm 2.7$ & $-2.8 \pm 0.7$ & - & - & - & - & - \\
\hline & & [34] (mm) & BFR & $11.2 \pm 2.6$ & $-2.1 \pm 0.9$ & - & - & - & - & - \\
\hline & & Hughes et al. & $\mathrm{EG}_{1}$ & - & $0.03 \pm 0.01$ & $0.12 \pm 0.06$ & - & - & - & - \\
\hline & & [47] (cm) & BFR & - & $0.02 \pm 0.01$ & $0.10 \pm 0.04$ & - & - & - & - \\
\hline & & & $\mathrm{EG}_{1}$ & - & - & $-3.1 \pm 3.5$ & - & - & - & - \\
\hline & \multirow{8}{*}{$\mathrm{TC}\left(\mathrm{mm}^{2}\right)$} & Curran et al. & $\mathrm{EG}_{2}$ & - & - & $-2.3 \pm 4.3$ & - & - & - & - \\
\hline & & {$[36]\left(\mathrm{cm}^{3}\right)$} & BFR & - & - & $-1.8 \pm 2.6$ & - & - & - & - \\
\hline & & & $\mathrm{EG}_{3}$ & - & - & $-1.5 \pm 2.4$ & - & - & - & - \\
\hline & & Ferraz et al. [44] & $\mathrm{EG}_{2}$ & $4600 \pm 950^{*}$ & - & $4700 \pm 950^{*}$ & - & - & - & - \\
\hline & & & BFR & $4650 \pm 825 *$ & - & $4950 \pm 750 *$ & - & - & - & - \\
\hline & & & $\mathrm{EG}_{1}$ & $4250 \pm 400 *$ & - & $4450 \pm 400 *$ & - & - & - & - \\
\hline & & Rodrigues et al. & BFR & $4200 \pm 225$ * & - & $4400 \pm 300^{*}$ & - & - & - & - \\
\hline & & & $\mathrm{CON}$ & $4350 \pm 450 *$ & - & $4375 \pm 425 *$ & - & - & - & - \\
\hline & \multirow{8}{*}{$\begin{array}{c}\text { Measuring } \\
\text { tape }(\mathrm{cm})\end{array}$} & Barbalho et al. & $\mathrm{CON}$ & $48.2 \pm 2.5$ & $-3.6 \pm 1.3$ & - & - & - & - & - \\
\hline & & [34] & BFR & $48.1 \pm 2.9$ & $-2.5 \pm 1.1 *$ & - & - & - & - & - \\
\hline & & Barbosa et al. & $\mathrm{CON}$ & $\begin{array}{c}25.62 \\
(23.67-27.56)\end{array}$ & - & $\begin{array}{c}25.84 \\
(24.11-27.57)\end{array}$ & - & - & - & - \\
\hline & & & BFR & $\begin{array}{c}26.27 \\
(24.87-27.67)\end{array}$ & - & $\begin{array}{c}26.49 \\
(25.13-27.85)\end{array}$ & - & - & - & - \\
\hline & & & & $(44-52) / 49.00$ & $6 \mathrm{~cm}-\mathrm{p}$ (i): 50.00 & & & & & \\
\hline & & Tennent et al. & $\mathrm{CON}$ & $(45.5-51)$ & $(45.5-50.5)$ & - & - & - & - & - \\
\hline & & [53] & & $\begin{array}{c}16 \mathrm{~cm}-\mathrm{p}(\mathrm{u} / \mathrm{i}): \\
59.50\end{array}$ & $\begin{array}{c}16 \text { cm-p (i): } \\
60.00(54-61)\end{array}$ & & & & & \\
\hline & & & & $\begin{array}{c}(53-62) / 60.00 \\
(54-61)\end{array}$ & & & & & & \\
\hline
\end{tabular}


Table 3. Cont.

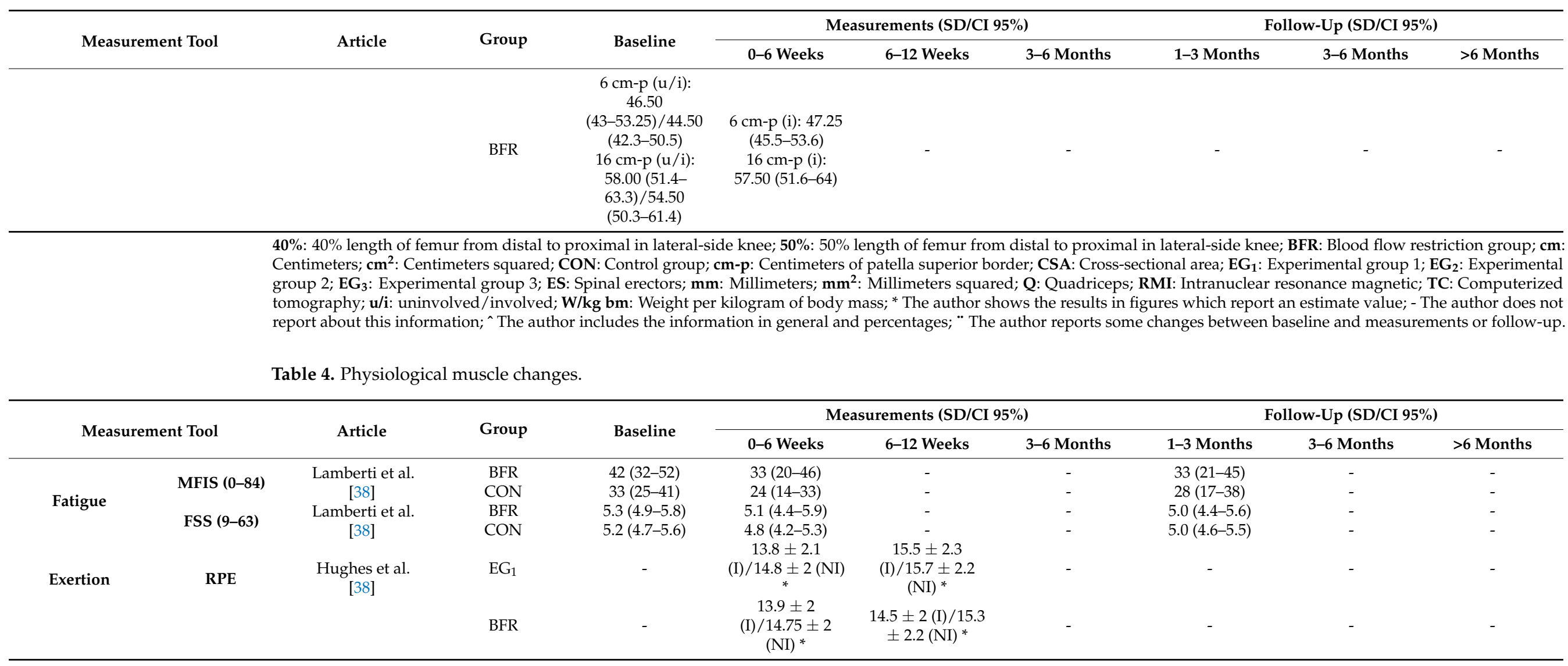


Table 4. Cont.

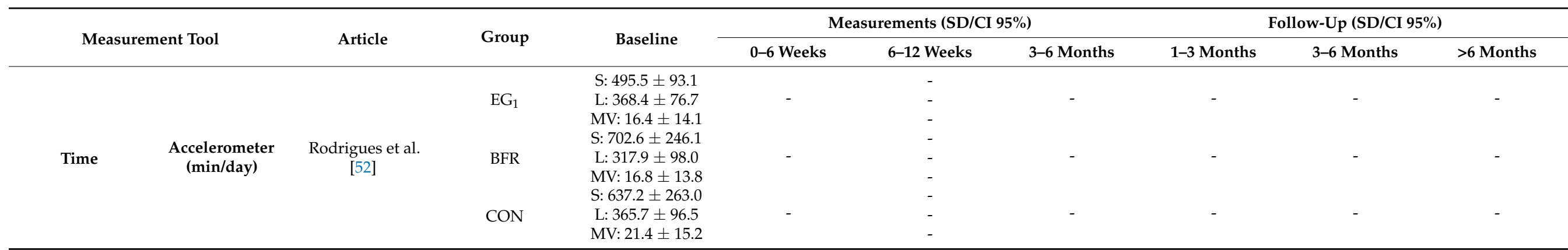

BFR: Blood flow restriction group; EG$_{1}$ : Experimental group 1; FSS: Fatigue severity scale; I: Injured limb; L: Light physical activity; min/day: Minutes per day; MFIS: Modified fatigue impact scale; MV: Moderate to vigorous activity; NI: Non-injured limb; RPE: Rating Perceived Exertion Scale; S: Sedentary activity; ${ }^{*}$ The author shows the results in figures which they report an estimate value.

Table 5. Blood/cardiocirculatory outcomes.

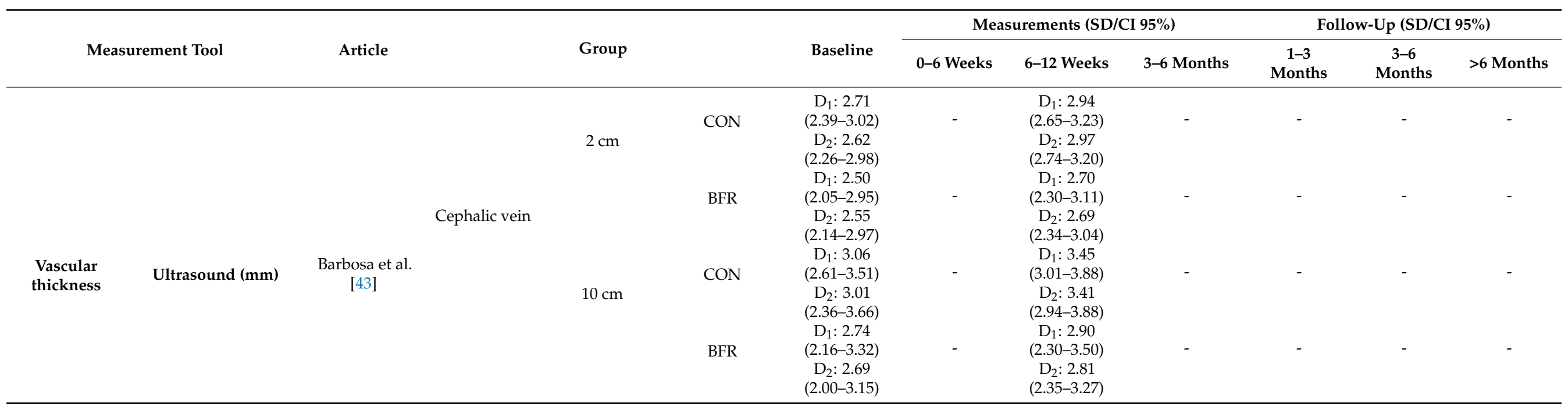


Table 5. Cont.

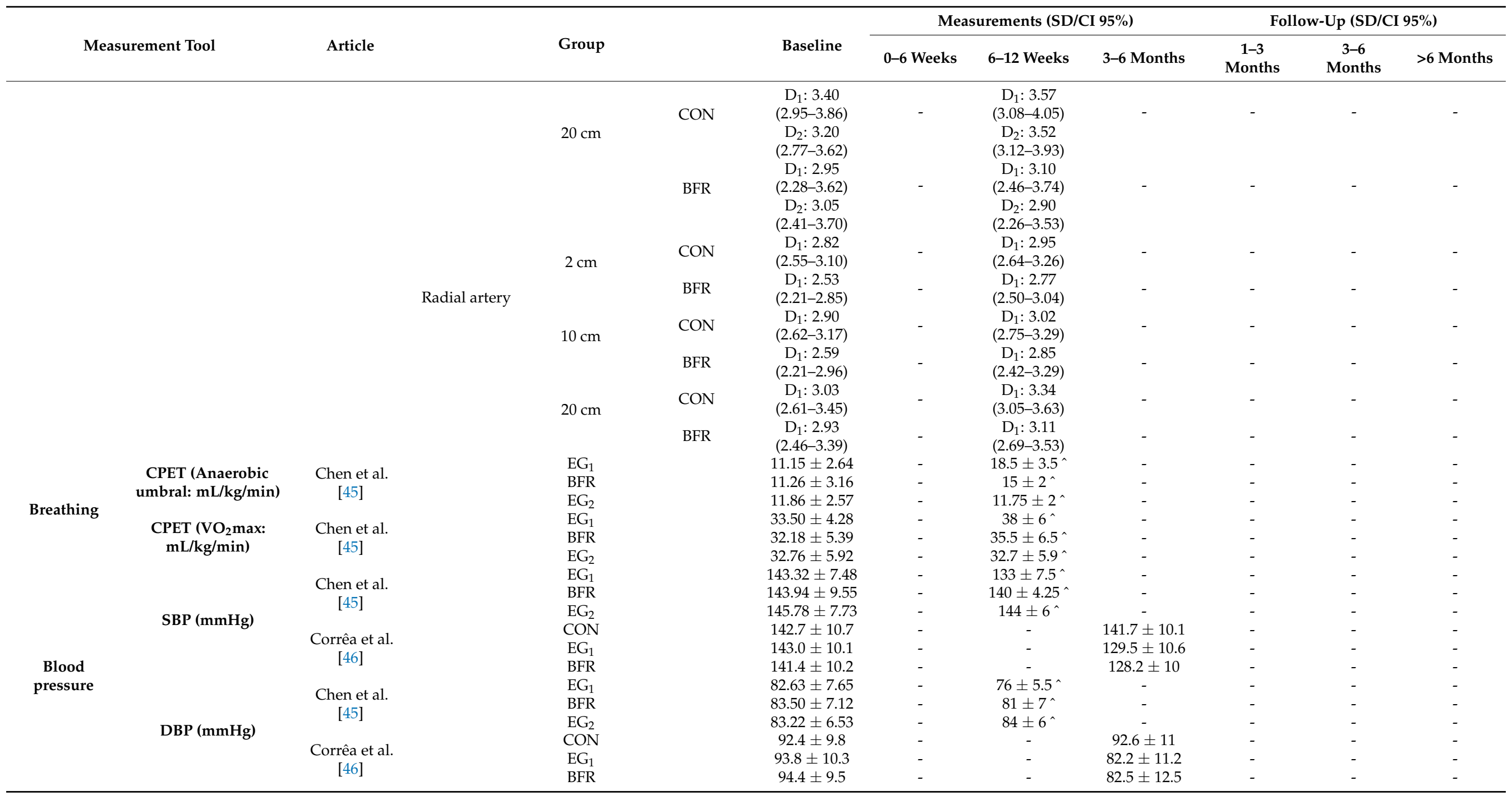


Table 5. Cont.

\begin{tabular}{|c|c|c|c|c|c|c|c|c|c|c|}
\hline \multirow{2}{*}{\multicolumn{2}{|c|}{ Measurement Tool }} & \multirow[b]{2}{*}{ Article } & \multirow[b]{2}{*}{ Group } & \multirow[b]{2}{*}{ Baseline } & \multicolumn{3}{|c|}{ Measurements (SD/CI 95\%) } & \multicolumn{3}{|c|}{ Follow-Up (SD/CI 95\%) } \\
\hline & & & & & 0-6 Weeks & 6-12 Weeks & 3-6 Months & $\begin{array}{c}1-3 \\
\text { Months }\end{array}$ & $\begin{array}{c}\text { 3-6 } \\
\text { Months }\end{array}$ & $>6$ Months \\
\hline \multirow{9}{*}{ Length } & \multirow{3}{*}{ ECG (LVEF: \%) } & \multirow{3}{*}{$\begin{array}{l}\text { Chen et al. } \\
\text { [45] }\end{array}$} & $\mathrm{EG}_{1}$ & $54.21 \pm 7.38$ & - & $61 \pm 4.5^{\wedge}$ & - & - & - & - \\
\hline & & & BFR & $53.39 \pm 7.41$ & - & $57.5 \pm 5.5^{\wedge}$ & - & - & & - \\
\hline & & & $\mathrm{EG}_{2}$ & $51.44 \pm 7.60$ & - & $52 \pm 7.25^{\wedge}$ & - & - & - & - \\
\hline & \multirow{3}{*}{ ECG (LVEDD: $\mathrm{mm}$ ) } & \multirow{3}{*}{$\begin{array}{c}\text { Chen et al. } \\
\text { [45] }\end{array}$} & $\mathrm{EG}_{1}$ & $47.53 \pm 7.31$ & - & $43.75 \pm 6.5^{\wedge}$ & - & - & - & - \\
\hline & & & BFR & $48.44 \pm 8.46$ & - & $45.5 \pm 7.75^{\wedge}$ & - & - & - & - \\
\hline & & & $\mathrm{EG}_{2}$ & $50.89 \pm 7.45$ & - & $50.4 \pm 7.5^{\wedge}$ & - & - & - & - \\
\hline & \multirow{3}{*}{ ECG (LVESD: mm) } & \multirow{3}{*}{$\begin{array}{c}\text { Chen et al. } \\
\text { [45] }\end{array}$} & $\mathrm{EG}_{1}$ & $35.68 \pm 6.54$ & - & $29.75 \pm 5.25^{\wedge}$ & - & - & - & - \\
\hline & & & BFR & $36.22 \pm 6.81$ & - & $33.5 \pm 6.5^{\wedge}$ & - & - & - & - \\
\hline & & & $\mathrm{EG}_{2}$ & $38.11 \pm 7.11$ & - & $38.5 \pm 6.25^{\wedge}$ & - & - & - & - \\
\hline
\end{tabular}

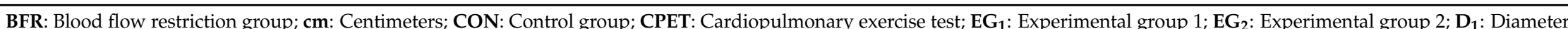
$\mathbf{D}_{2}$ : Distensibility; ECG: Electrocardiogram; LVEDD: Left ventricular end-diastolic dimension; LVESD: Left ventricular end-systolic dimension; LVEF: left ventricular ejection fraction; $\mathbf{m m}$ : Millimeters; $\mathbf{m L} / \mathbf{k g} / \mathbf{m i n}$ : millimeters per kilogram per minute; $\mathbf{V O}_{2}$ max: Maximum volume of oxygen; * The author shows the results in figures which they report an estimate value; ^ The author includes the information in general and percentages. 


\section{Discussion}

In this review, 20 clinical trials were included, in which the effectiveness of the BFR tool was evaluated together with other exercise methodologies on variables such as strength, muscular structural changes, muscular physiological changes and cardiovascular variables in blood in neuro-musculoskeletal patients. A disparity in the results is found among the observed findings, in which the BFR becomes an alternative tool to high-load exercises in the short and medium term, since the neuromuscular and hormonal reactions caused by the pressure promote the segregation of insulin-like growth factor 1 (IGF-1) and an increase in muscle activation [8]. Some specific aspects are analyzed below.

\subsection{Strength}

For the strength variable (Table 2), heterogeneity is shown in terms of the data collection unit, especially with the dynamometer measurement tool. In the short term, changes in the dynamometer are evidenced in favor of the control groups with $2.3 \mathrm{Nm}$ [38] and the experimental groups with $7.1 \mathrm{Nm}$ [41]. In both studies, the study subjects presented the same pathology and better results when they performed moderate-intensity or lowload exercises. This finding may be since they use different pressure-applying methods with the cuff to the usual protocol, or because patients with knee osteoarthritis present a more complicated clinical picture due to the presence of central sensitization, requiring a multidisciplinary approach [54].

Meanwhile, in one study, changes were observed in favor of the control group for each of the movements in the uninjured leg with average values of 1.9 and 4.1 newtonmeter split kilogram $(\mathrm{Nm} / \mathrm{kg})$, except for the uncorrected extension, where the BFR group improved $9 \mathrm{Nm} / \mathrm{kg}$ more than the control group. For the injured leg, the BFR group presented differences in all movements between 14.6 and $66.2 \mathrm{Nm} / \mathrm{kg}$, although in deficit, the control group found lower differences between both limbs with values between -1.12 and $-33.44 \mathrm{Nm} / \mathrm{kg}$ [50]. Similarly, patients who received cardiac surgery were able to reduce the loss of strength in the BFR group with an average deficit of $1.2 \mathrm{~kg}$-force for knee extension and fist closure compared to the control group [55]. These between-group differences may be due to the fact that the BFR group uses resistance exercises compared to the control group; therefore, it can be suggested that knee arthroscopy and cardiac surgery patients may benefit from the application of BFR together with resistance exercises; strength, rather than aerobic exercise; or exercise without a pattern of progression [49].

According to the 1RM test, benefits are found for the control group of $2.2 \mathrm{~kg}$ [41], while in the $10 \mathrm{RM}$ test, changes are observed for the BFR group in both the uninjured leg with a $0.23 \mathrm{~kg}$ match body mass $(\mathrm{kg} / \mathrm{kg} \mathrm{bm})$ as in the injured leg with $0.305 \mathrm{~kg} / \mathrm{kg} \mathrm{bm}$ [56]. The differences between the patient profiles of each study are minimal; therefore, regardless of using/not using low-load exercise with or without BFR, the same benefits are generated as in the case of the first study. Furthermore, in the second study, the patients improved the strength of both lower limbs, which could be a possible consequence related to the application of greater metabolic stress to replace mechanical stress [7].

The dynamometer tool shows favorable differences for all groups in the medium term, especially for the BFR group, with improvement changes between $11.52 \%$ and $26.83 \%[36,38,43]$ and between $-8.57 \%$ and $9.09 \%$ in the entire knee flexion-extension range on both the unaffected and injured sides, except for the unaffected side at 60 degrees with $14.63 \%$ and at 300 degrees with $12.12 \%$, where there was greater improvement in the high-load exercise group [56]. In addition, for patients with cardiac surgery, in the BFR group, it was observed that knee extension strength improved by $36.7 \%$ and fist closure by $12.89 \%$ [55]. These benefits of the BFR tool explain its usefulness with different exercise methodologies, such as aerobic and strength; in different knee pathologies; or in renal and cardiac pathologies. In addition, pressures between $50 \%$ and $80 \%$ of the maximum arterial occlusion with the cuff were used at all times, suggesting that the same results would be obtained regardless of this variable [9]. 
In contrast, there are studies that demonstrate improvements, such as control and experimental improvements, that show changes between $-8.1 \%$ and $9.34 \%$ without statistically significant differences with respect to the BFR group [37,44], with the exception of one study where the control group showed an improvement of $8.4 \%$, i.e., almost double that of the BFR group [42]. The dissonance of this study's results, with respect to the remaining analyzed studies, may be due to the fact that the BFR group performed the exercise with different parameters to the established protocol for its application, using different maximum repetition percentages for the concentric and eccentric phases of the exercise, as well as the execution time used in each phase of the exercise [13].

For the 1RM tool, the BFR group improved strength by $2.94 \%$ [37], although in movements such as knee extension and leg press, the high-load exercise group obtained changes of between 22.85 and $28 \%$; $78 \%$ without benefits superior to the BFR group [46,47], which was better in knee extension with a change of $28.78 \%$ [48]. Both the BFR and experimental groups improved equally, meaning that high-load and low-load exercise combined with BFR provided the same benefits for subjects with knee pathologies. Greater benefits were found only in one study when applying BFR in older people, which may suggest that this tool is ideal for introducing adequate fatigue that low-load exercise alone cannot provide [57].

In relation to other evaluation tools, changes of $91.15 \%$ were found for the injured side and $40.77 \%$ for the non-injured side when evaluated with the 10RM after performing high-load exercises [56]. In addition, a difference of $0.2 \%$ in the central activation ratio was observed for experimental group 3, which used 70\% BFR during the concentric phase and $20 \%$ during the eccentric phase of the 1RM in the leg press [37]. The evaluation was not considered statistically significant in either of the two tools with respect to the other groups; however, it indicates that the BFR group improved in the 10RM by inducing metabolic stress [57] and in the case of the central activation ratio, since the combination of eccentric exercise and BFR cuff pressure cause corticalization of the muscle being exercised [58].

Likewise, only one study was found to assess the effects of the BFR tool, where together with the exercise group with moderate loads the same benefits were obtained, although the latter showed a greater difference of $6.5 \%$ than the BFR group [53]. These benefits suggest that low-load exercises, together with BFR or moderate loads, can produce the same benefits in patients with chronic kidney disease in phase 2 . Therefore, it is noted that applying pressure with the BFR tool obtains good results in terms of strength as a possible consequence of improving patient hemodynamics [40].

Regarding the follow-up, the dynamometer tool demonstrates changes in favor of the control group with $14.3 \%$ in subjects with recurrent non-specific lumbar pain [42]. Although there are no major changes compared to the BFR group, it should be noted that this study's methodology is not well-planned from a treatment point of view; rather, it seeks to observe the cross-transfer effect of the BFR, which does not seem to be provoked but is directly associated with the presence of metabolites [59].

Similarly, the control group shows significant benefits for $4.41 \%$ for men with osteoarthritis [41], although the same treatment plan led to better results for women in the BFR group, with a change of 5.38\% [40]. In turn, it is extrapolated in the 1RM evaluation, where men in the control group improved by $34.81 \%$ and women in the BFR group improved by $17.39 \%$, even $4.92 \%$ in $40 \%$ of $1 \mathrm{RM}$. These results may suggest that men with osteoarthritis are better adapted to low-load work, while women do so through the use of BFR.

Finally, the BFR group obtained better results in the Manual Muscle Test- 8 with an increase of $1.28 \%$, while in the kinetic communicator tool, no changes were found with respect to the evaluation, unlike the control group with negative results [48]. These minor differences shown - with respect to the control group without considerably increasing the benefits-may be due to the use of a random pressure which is not controlled by vascular Doppler where the degree of arterial occlusive pressure can be evaluated, similarly to when the elastic bands are applied [60]. 


\subsection{Structural Muscle Changes}

Continuing with the structural change variable shown in Table 3, in the short term, improvements were observed for the muscle cross-sectional area by intranuclear magnetic resonance and ultrasound of between $-12.65 \%$ [57] and $-18.75 \%$ for the BFR group [35], $-13.88 \%$ for the control group [49] and $0.03 \%$ for the high-load exercise group [56]. This heterogeneity in the results is close to that of the comparative groups; therefore, BFR combined with low-load exercise with passive mobilizations stands as a tool together with high-load exercise to prevent the loss of muscle mass in patients with muscle reconstruction, anterior cruciate ligaments and older adults in a coma [61]. On the other hand, when evaluating muscle thickness using a tape measure, improvements ranging between $-5.2 \%$ and $6.18 \%$ were found $[49,50]$. In this case, patients with nonreconstructive knee arthroscopy and older adults in a coma could benefit from low-load exercises or passive mobilizations applied together with BFR compared to doing nothing or day-to-day activities as a possible consequence of metabolic stress when submitting [7].

In the medium term, both experimental group 1-which used high-load exercises, with an average of $4.1 \%[36,46,56]$ as well as the BFR-and experimental group 3 improved the muscle cross-sectional area, the latter applying restriction of blood flow with different percentages of maximum repetition in the concentric and eccentric phases, with a mean of $1.63 \%[37,47]$. The difference in the results may be due to the use of different evaluation tools such as magnetic resonance imaging, ultrasound and computed tomography, although this difference is significantly small between the experimental and BFR groups. It can also be said that it is a favorable treatment tool for pathologies such as knee or systemic diseases that are both valid in the recovery of muscle mass. This disparity in results was also significantly present in a study where the control group prevented the loss of muscle mass in the erector spinae with $-1.7 \%$ and the BFR group increased the muscle mass of the quadriceps by $2.9 \%$ [42]. In addition, when evaluating muscle thickness with a gold measuring tape, it was found that the control group obtained better results with $0.86 \%$ [44]. Therefore, it is suggested that the application of the cuff generates an increase in, or prevention of, loss of muscle mass to the applied area [62].

Regarding the long term, no data is collected. Only in short-term follow-up were increases in muscle mass observed for the BFR groups of $1.92 \%$ on average [41,42] and for the control group of $2.5 \%$ in the erector spinae [42]. This indicates that post-intervention results continue to be highly beneficial for patients with recurrent nonspecific low back pain and knee osteoarthritis in men, and may be associated with improvements in their quality of life and functionality $[63,64]$

\subsection{Physiological Muscle Changes}

Within the muscle physiological changes, short-term changes were observed for the fatigue variable in the BFR group of $21.43 \%$ with the Modified Fatigue Impact Scale (MFIS) and in the control group of $7.69 \%$ with the Fatigue Severity Scale (FSS) [39]. These results confirm that the application of BFR while walking produces the same benefits as walking, although the difference-in favor of the BFR group-may be due to the fact that a lower pressure than usual was applied in the control group [10]. On the other hand, in the effort variable, no differences were found between the BFR groups and high-load exercise for both legs, showing changes in the Rating Perceived of Exertion (RPE) of $13.9 \%$ for the BFR group in the injured limb and $14 \%$ and $8 \%$ for the control group in the injured leg [51]. This finding may indicate that the BFR tool, together with low-load exercises, can achieve the same levels of effort as high-load exercises and may be due to metabolic stress [57].

In relation to the medium term, the experimental group obtained better results than the BFR group in the effort variable, with an average of $15.6 \%$ for both members, both healthy and injured [51]. However, the results of the BFR group are similar to those of the experimental group, so it cannot be corroborated that high-load exercises improve fatigue perception more than the low-load exercise group with BFR. An important point in the 
generation of this greater fatigue may be due to the application of an arterial occlusive pressure of $80 \%$, so that the oxygen supply to the tissues may be lower [61].

In the long term, only one study evaluated the fatigue variable, where significant results were found for the BFR group in the MFIS and FSS, with changes of $21.43 \%$ and $5.66 \%$, respectively [39]. In this case, the BFR group may not have generated much fatigue due to the fact that they carried out a different method from the common one, such as intermittent pressure with the cuff, which may reduce this sensation [13].

\subsection{Hemodynamic Variables and Vascular Caliber}

Regarding vascular thickness, all measurements were collected in the medium term. For the vascular thickness of the cephalic vein, the control group obtained better results with the electrocardiogram in measurements of 2, 10 and $20 \mathrm{~cm}$-both for the diameter, with a mean of $8.75 \%$; and for the compliance, with an average of $12.22 \%$. Moreover, the thickness at the cephalic artery level varies minorly, with an average of $9.76 \%$ in 2 and $10 \mathrm{~cm}$ for the BFR group and in $20 \mathrm{~cm}$ for the control group with 10.23\% [44]. This difference within the vascular system may be associated with the fact that BFR only acts directly on the arterial system instead of the venous system, hence only improvements in the cephalic artery are observed when used with low-load exercise [65].

Finally, one study assesses the subvariables of respiration, blood pressure and length, showing changes in favor of the group of isometric exercises plus medication intake for the anaerobic threshold of $65.91 \%$, a maximum oxygen volume of $13.43 \%$, ejection fraction of the left ventricle of $6.79 \%$ and a final diastolic dimension of the left ventricle of $1.02 \%$ [52] In relation to systolic and diastolic blood pressure, in both isometric exercise groups, moderate loads and BFR obtained similar parameters: around $8.32 \%$ on average for systolic blood pressure and $10.31 \%$ for diastolic blood pressure [52,53]. However, the group that only received medication obtained greater benefits in the final systolic dimension of the left ventricle with $-0.96 \%$ [52]. One of the reasons why the isometric exercise group obtained better results than the rest of the groups might be that it is the only group which practiced exercise at a moderate intensity, while the BFR group practiced a protocol of inflating and deflating the cuff without exercising. Therefore, all vascular benefits of the BFR cuff may have been displaced, such as improving blood pressure levels [66].

\subsection{BFR: Clinical and Trainning Consideration}

In view of the clinical applicability of the results observed in the present study, it is important to identify the outcome variable that is intended to be improved when restricting blood flow. In this sense, it was observed how the functional variables undergo a statistically significant improvement in the short and medium term when a moderate blood flow restriction is performed, i.e., $50-60 \%$, while there is more controversy when the blood flow restriction is higher than this percentage or when the pressure exerted is similar for all participants in absolute terms.

On the other hand, in order to obtain structural changes in the muscle in the shortmedium term, only significant changes were observed in the diameter of the brachial artery, while in the rest of the structural variables, there is considerable controversy regarding the changes generated, since in some cases it is better than the control group, but equal to or worse than some of the experimental groups that do not have blood flow restriction as a complement to training. In this sense, it may be necessary to carry out longer clinical trials, since the structural changes begin to consolidate in the medium long term, and the improvements observed at the functional level may be the consequence of better efficiency of the motor units, accompanied in the medium term by an increase in muscular vascularization. It would therefore be advisable to design and carry out randomized clinical trials with an analysis of the evolution of the structural characteristics in the medium and long term. 
On the other hand, considering the objective of the use of blood flow restriction, i.e., rehabilitation or training, the fact that there is a functional improvement with moderate BFR intensities allows the person being rehabilitated to obtain an increase in function in the short term, which can be key in the first stages of the beginning of recovery since it allows a functional improvement in the short and medium term using moderate blood flow restrictions along with moderate contraction intensities, creating a perfect scenario for those who temporarily experience a reduction in their functional capacity. On the other hand, a longer period of training is required to achieve structural adaptations, so it would be necessary to increase the number of clinical trials aimed at generating structural changes in the muscle. Although it is observed that for training, more intense blood flow restrictions, generating vascular ischemia, or a progressive increase in the intensity of flow restriction seem to have promising results when it comes to training and favoring structural changes.

\section{Conclusions}

The main conclusions that can be reached from carrying out this systematic review are that the application of the BFR technique can achieve benefits in the short and medium term to increase strength, muscle thickness and cardiovascular endurance, as well as improving the physiological level of the cardiovascular system. In addition, BFR combined with low-load exercises also achieves benefits comparable to high-intensity exercises without the application of BFR, benefiting patients who are unable to lift high loads. Some reasons which support these results are the replacement of mechanical stress by metabolic stress and the corticomotor activation of the muscle. However, the results of the included studies are insufficient to indicate a favorable trend from the effects of BFR in the long term and follow-up; therefore, future studies are required to evaluate these periods in order to demonstrate whether its effects are maintained or improved with respect to high-load or aerobic exercises.

Author Contributions: Conceptualization, Á.J.R.-R., G.M.-T., R.P.R.-G. and M.G.-S.; methodology, Á.J.R.-R., A.G.-M.; G.M.-T., J.A.M.-B., R.P.R.-G. and M.G.-S.; validation, Á.J.R.-R., A.G.-M., J.A.M.-B. and R.P.R.-G.; formal analysis, Á.J.R.-R. and R.P.R.-G.; investigation, Á.J.R.-R. and A.G.-M.; resources, Á.J.R.-R. and J.A.M.-B.; data curation, Á.J.R.-R., J.A.M.-B. and R.P.R.-G.; writing-original draft preparation, Á.J.R.-R., A.G.-M.; G.M.-T., J.A.M.-B., R.P.R.-G. and M.G.-S.; writing-review and editing, Á.J.R.-R., A.G.-M., G.M.-T., J.A.M.-B., R.P.R.-G. and M.G.-S.; supervision, A.G.-M., G.M.-T., J.A.M.-B. and M.G.-S.; project administration, A.G.-M., G.M.-T. and M.G.-S. All authors have read and agreed to the published version of the manuscript.

Funding: Erasmus+ Strategic Partnership for Higher Education Program: 2018-1-PL01-KA203-051055.

Institutional Review Board Statement: Not applicable.

Informed Consent Statement: Not applicable.

Conflicts of Interest: The authors declare no conflict of interest.

\section{References}

1. Anderson, A.B.; Owens, J.G.; Patterson, S.D.; Dickens, J.F.; LeClere, L.E. Blood flow restriction therapy: From development to applications. Sports Med. Arthrosc. Rev. 2019, 27, 119-123. [CrossRef]

2. Pignanelli, C.; Christiansen, D.; Burr, J.F. Blood flow restriction training and the high-performance athlete: Science to application. J. Appl. Physiol. 2021, 130, 1163-1170. [CrossRef]

3. Bielitzki, R.; Behrendt, T.; Behrens, M.; Schega, L. Time to Save Time: Beneficial Effects of Blood Flow Restriction Training and the Need to Quantify the Time Potentially Saved by its Application during Musculoskeletal Rehabilitation. Phys. Ther. 2021, 101, pzab172. [CrossRef] [PubMed]

4. Patterson, S.D.; Hughes, L.; Warmington, S.; Burr, J.; Scott, B.; Owens, J.; Abe, T.; Nielsen, J.; Libardi, C.A.; Laurentino, G.; et al. Blood Flow Restriction Exercise: Considerations of Methodology, Application, and Safety. Front. Physiol. 2019, 10, 533. [CrossRef]

5. Buckner, S.L.; Dankel, S.J.; Counts, B.R.; Jessee, M.B.; Mouser, J.G.; Mattocks, K.T.; Laurentino, G.C.; Abe, T.; Loenneke, J.P. Influence of cuff material on blood flow restriction stimulus in the upper body. J. Physiol. Sci. 2017, 67, 207-215. [CrossRef]

6. Slysz, J.; Stultz, J.; Burr, J.F. The efficacy of blood flow restricted exercise: A systematic review \& meta-analysis. J. Sci. Med. Sport 2016, 19, 669-675. [PubMed] 
7. Pearson, S.J.; Hussain, S.R. A Review on the Mechanisms of Blood-Flow Restriction Resistance Training-Induced Muscle Hypertrophy. Sports Med. 2015, 45, 187-200. [CrossRef]

8. Heitkamp, H.C. Training with blood flow restriction. Mechanisms, gain in strength and Safety. J. Sports Med. Phys. Fit. 2015, 55, 446-456.

9. Manini, T.M.; Vincent, K.R.; Leeuwenburgh, C.L.; Lees, H.A.; Kavazis, A.N.; Borst, S.E.; Clark, B.C. Myogenic and proteolytic mRNA expression following blood flow restricted exercise. Acta Physiol. 2011, 201, 255-263. [CrossRef] [PubMed]

10. Hughes, L.; Paton, B.; Rosenblatt, B.; Gissane, C.; Patterson, S.D. Blood flow restriction training in clinical musculoskeletal rehabilitation: A systematic review and meta-analysis. Br. J. Sports Med. 2017, 51, 1003-1011. [CrossRef]

11. Clarkson, M.J.; May, A.K.; Warmington, S.A. Is there rationale for the cuff pressures prescribed for blood flow restriction exercise? A systematic review. Scand. J. Med. Sci. Sports 2020, 30, 1318-1336. [CrossRef]

12. Centner, C.; Lauber, B. A Systematic Review and Meta-Analysis on Neural Adaptations Following Blood Flow Restriction Training: What We Know and What We Don't Know. Front. Physiol. 2020, 11, 887. [CrossRef]

13. Lu, Y.; Patel, B.H.; Kym, C.; Nwachukwu, B.U.; Beletksy, A.; Forsythe, B.; Chahla, J. Perioperative Blood Flow Restriction Rehabilitation in Patients Undergoing ACL Reconstruction: A Systematic Review. Orthop. J. Sports Med. 2020, 8, 2325967120906822. [CrossRef] [PubMed]

14. Van Cant, J.; Dawe-Coz, A.; Aoun, E.; Esculier, J.F. Quadriceps strengthening with blood flow restriction for the rehabilitation of patients with knee conditions: A systematic review with meta-analysis. J. Back Musculoskelet. Rehabil. 2020, 33, 529-544. [CrossRef]

15. Cerqueira, M.S.; Do Nascimento, J.D.S.; Maciel, D.G.; Barboza, J.A.M.; Vieira, W.H.D.B. Effects of blood flow restriction without additional exercise on strength reductions and muscular atrophy following immobilization: A systematic review: Passive blood flow restriction and disuse. J. Sport Health Sci. 2020, 9, 152-159. [CrossRef] [PubMed]

16. Grønfeldt, B.M.; Nielsen, J.L.; Mieritz, R.M.; Lund, H.; Aagaard, P. Effect of blood-flow restricted vs heavy-load strength training on muscle strength: Systematic review and meta-analysis. Scand. J. Med. Sci. Sports 2020, 30, 837-848. [CrossRef]

17. Centner, C.; Wiegel, P.; Gollhofer, A.; König, D. Effects of Blood Flow Restriction Training on Muscular Strength and Hypertrophy in Older Individuals: A Systematic Review and Meta-Analysis. Sports Med. 2019, 49, 95-108. [CrossRef]

18. Formiga, M.F.; Fay, R.; Hutchinson, S.; Locandro, N.; Ceballos, A.; Lesh, A.; Buscheck, J.; Meanor, J.; Owens, J.G.; Cahalin, L.P. Effect of Aerobic Exercise Training With and Without Blood Flow Restriction on Aerobic Capacity in Healthy Young Adults: A Systematic Review With Meta-Analysis. Int. J. Sports Phys. Ther. 2020, 15, 175-187. [CrossRef] [PubMed]

19. Silva, J.C.G.; Pereira Neto, E.A.; Pfeiffer, P.A.S.; Neto, G.R.; Rodrigues, A.S.; Bemben, M.G.; Patterson, S.D.; Batista, G.R.; Cirilo-Sousa, M.S. Acute and Chronic Responses of Aerobic Exercise With Blood Flow Restriction: A Systematic Review. Front. Physiol. 2019, 10, 1239. [CrossRef]

20. Neto, G.R.; Novaes, J.S.; Dias, I.B.F.; Brown, A.; Vianna, J.; Cirilo-Sousa, M.S. Effects of resistance training with blood flow restriction on haemodynamics: A systematic review. Clin. Physiol. Funct. Imaging 2017, 37, 567-574. [CrossRef] [PubMed]

21. Domingos, E.; Polito, M.D. Blood pressure response between resistance exercise with and without blood flow restriction: A systematic review and meta-analysis. Life Sci. 2018, 209, 122-131. [CrossRef]

22. Loenneke, J.P.; Wilson, J.M.; Marín, P.J.; Zourdos, M.C.; Bemben, M.G. Low intensity blood flow restriction training: A metaanalysis. Eur. J. Appl. Physiol. 2012, 112, 1849-1859. [CrossRef] [PubMed]

23. Lixandrão, M.E.; Ugrinowitsch, C.; Berton, R.; Vechin, F.; Conceição, M.S.; Damas, F.; Libardi, C.A.; Roschel, H. Magnitude of Muscle Strength and Mass Adaptations Between High-Load Resistance Training Versus Low-Load Resistance Training Associated with Blood-Flow Restriction: A Systematic Review and Meta-Analysis. Sports Med. 2018, 48, 361-378. [CrossRef] [PubMed]

24. Da Cunha Nascimento, D.; Petriz, B.; da Cunha Oliveira, S.; Vieira, D.C.L.; Funghetto, S.S.; Silva, A.O.; Prestes, J. Effects of blood flow restriction exercise on hemostasis: A systematic review of randomized and non-randomized trials. Int. J. Gen. Med. 2019, 12, 91-100. [CrossRef]

25. Baker, B.; Stannard, M.S.; Duren, D.L.; Cook, J.L.; Stannard, J.P. Does Blood Flow Restriction Therapy in Patients Older Than Age 50 Result in Muscle Hypertrophy, Increased Strength, or Greater Physical Function? A Systematic Review. Clin. Orthop. Relat. Res. 2020, 478, 593-606. [CrossRef]

26. Clarkson, M.; May, A.; Warmington, S.A. Chronic Blood Flow Restriction Exercise Improves Objective Physical Function: A Systematic Review. Front. Physiol. 2019, 10, 1058. [CrossRef]

27. Maher, C.G.; Sherrington, C.; Herbert, R.D.; Moseley, A.M.; Elkins, M. Reliability of the PEDro Scale for Rating Quality of Randomized. Phys. Ther. 2003, 83, 713-721. [CrossRef]

28. Escobar Calderón, E.H.; Ramos Bazán, A.G. Efectividad de un Programa de Entrenamiento Físico Para Mejorar la Fuerza Muscular de los Pacientes Adultos en Hemodiálisis; Universidad Norbert Wiener: Lima District, Peru, 2020.

29. NLM. Muscle Strength. In Medical Subject Headings; National Library of Medicine: Bethesda, MD, USA, 2007.

30. Brown, S.C.; Sewry, C.A. Basics of Skeletal Muscle Function and Normal Physiology. In Cardioskeletal Myopathies in Children and Young Adults; Elsevier Inc.: Amsterdam, The Netherlands, 2017; pp. 21-38. [CrossRef]

31. Jones, E.J.; Bishop, P.A.; Woods, A.K.; Green, J.M. Cross-sectional area and muscular strength: A brief review. Sports Med. 2008, 38, 987-994. [CrossRef]

32. Pisanu, S.; Deledda, A.; Loviselli, A.; Huybrechts, I.; Velluzzi, F. Validity of Accelerometers for the Evaluation of Energy Expenditure in Obese and Overweight Individuals: A Systematic Review. J. Nutr. Metab. 2020, 2020, 2327017. [CrossRef]

33. Instituto Nacional del Cáncer. Análisis de Sangre; Instituto Nacional del Cáncer-NIH: Bethesda, MD, USA, 2019. 
34. Barbalho, M.; Rocha, A.C.; Seus, T.L.; Raiol, R.; Del Vecchio, F.B.; Coswig, V.S. Addition of blood flow restriction to passive mobilization reduces the rate of muscle wasting in elderly patients in the intensive care unit: A within-patient randomized trial. Clin. Rehabil. 2019, 33, 233-240. [CrossRef] [PubMed]

35. Giles, L.; Webster, K.E.; McClelland, J.; Cook, J.L. Quadriceps strengthening with and without blood flow restriction in the treatment of patellofemoral pain: A double-blind randomised trial. Br. J. Sports Med. 2017, 51, 1688-1694. [CrossRef]

36. Curran, M.T.; Bedi, A.; Mendias, C.L.; Wojtys, E.M.; Kujawa, M.V.; Palmieri-Smith, R.M. Blood Flow Restriction Training Applied With High-Intensity Exercise Does Not Improve Quadriceps Muscle Function After Anterior Cruciate Ligament Reconstruction: A Randomized Controlled Trial. Am. J. Sports Med. 2020, 48, 825-837. [CrossRef]

37. Harper, S.A.; Roberts, L.M.; Layne, A.S.; Jaeger, B.C.; Gardner, A.K.; Sibille, K.T.; Wu, S.S.; Vincent, K.R.; Fillingim, R.B.; Manini, T.M.; et al. Blood-Flow Restriction Resistance Exercise for Older Adults with Knee Osteoarthritis: A Pilot Randomized Clinical Trial. J. Clin. Med. 2019, 8, 265. [CrossRef]

38. Lamberti, N.; Straudi, S.; Donadi, M.; Tanaka, H.; Basaglia, N.; Manfredini, F. Effectiveness of blood flow-restricted slow walking on mobility in severe multiple sclerosis: A pilot randomized trial. Scand. J. Med. Sci. Sports 2020, 30, 1999-2009. [CrossRef] [PubMed]

39. Segal, N.A.; Williams, G.N.; Davis, M.C.; Wallace, R.B.; Mikesky, A.E. Efficacy of Blood Flow-Restricted, Low-Load Resistance Training in Women with Risk Factors for Symptomatic Knee Osteoarthritis. PmE r 2015, 7, 376-384.

40. Segal, N.; Davis, M.D.; Mikesky, A.E. Efficacy of Blood Flow-Restricted Low-Load Resistance Training For Quadriceps Strengthening in Men at Risk of Symptomatic Knee Osteoarthritis. Geriatr. Orthop. Surg. Rehabil. 2015, 6, 160-167. [CrossRef]

41. Ampomah, K.; Amano, S.; Wages, N.P.; Volz, L.; Clift, R.; Ludin, A.F.M.; Nakazawa, M.; Law, T.D.; Manini, T.M.; Thomas, J.S.; et al. Blood Flow-restricted Exercise Does Not Induce a Cross-Transfer of Effect: A Randomized Controlled Trial. Med. Sci. Sports Exerc. 2019, 51, 1817-1827. [CrossRef]

42. Cardoso, R.K.; Araujo, A.M.; Del Vechio, F.B.; Bohlke, M.; Barcellos, F.C.; Oses, J.P.; de Freitas, M.P.; Rombaldi, A.J. Intradialytic exercise with blood flow restriction is more effective than conventional exercise in improving walking endurance in hemodialysis patients: A randomized controlled trial. Clin. Rehabil. 2020, 34, 91-98. [CrossRef]

43. Barbosa, J.B.; Maia, T.O.; Alves, P.S.; Bezerra, S.D.; Moura, E.C.; Medeiros, A.I.C.; Fuzari, H.K.; Rocha, L.G.; Marinho, P.E. Does blood flow restriction training increase the diameter of forearm vessels in chronic kidney disease patients? A randomized clinical trial. J. Vasc. Access 2018, 19, 626-633. [CrossRef]

44. Ferraz, R.B.; Gualano, B.; Rodrigues, R.; Kurimori, C.O.; Fuller, R.; Lima, F.R.; De Sá-Pinto, A.L.; Roschel, H. Benefits of Resistance Training with Blood Flow Restriction in Knee Osteoarthritis. Med. Sci. Sports Exerc. 2018, 50, 897-905. [CrossRef]

45. Chen, W.; Ni, J.; Qiao, Z.; Wu, Y.; Lu, L.; Zheng, J.; Chen, R.; Lu, X. Comparison of the clinical outcomes of two physiological ischemic training methods in patients with coronary heart disease. Open Med. 2019, 14, 224-233. [CrossRef]

46. Corrêa, H.L.; Neves, R.V.P.; Deus, L.A.; Maia, B.C.H.; Maya, A.T.; Tzanno-Martins, C.; Souza, M.K.; Silva, J.A.B.; Haro, A.S.; Costa, F.; et al. Low-load resistance training with blood flow restriction prevent renal function decline: The role of the redox balance, angiotensin 1-7 and vasopressin. Physiol. Behav. 2021, 230, 113295. [CrossRef]

47. Hughes, L.; Rosenblatt, B.; Haddad, F.; Gissane, C.; McCarthy, D.; Clarke, T.; Ferris, G.; Dawes, J.; Paton, B.; Patterson, S.D. Comparing the Effectiveness of Blood Flow Restriction and Traditional Heavy Load Resistance Training in the Post-Surgery Rehabilitation of Anterior Cruciate Ligament Reconstruction Patients: A UK National Health Service Randomised Controlled Trial. Sports Med. 2019, 49, 1787-1805. [CrossRef]

48. Hughes, L.; Patterson, S.D.; Haddad, F.; Rosenblatt, B.; Gissane, C.; McCarthy, D.; Clarke, T.; Ferris, G.; Dawes, J.; Paton, B. Examination of the comfort and pain experienced with blood flow restriction training during post-surgery rehabilitation of anterior cruciate ligament reconstruction patients: A UK National Health Service trial. Phys. Ther. Sport 2019, 39, 90-98. [CrossRef] [PubMed]

49. Iversen, E.; Røstad, V.; Larmo, A. Intermittent blood flow restriction does not reduce atrophy following anterior cruciate ligament reconstruction. J. Sport Health Sci. 2016, 5, 115-118. [CrossRef] [PubMed]

50. Jørgensen, A.N.; Aagaard, P.; Frandsen, U.; Boyle, E.; Diederichsen, L.P. Blood-flow restricted resistance training in patients with sporadic inclusion body myositis: A randomized controlled trial. Scand. J. Rheumatol. 2018, 47, 400-409. [CrossRef]

51. Ogawa, H.; Nakajima, T.; Shibasaki, I.; Nasuno, T.; Kaneda, H.; Katayanagi, S.; Ishizaka, H.; Mizushima, Y.; Uematsu, A.; Yasuda, T.; et al. Low-Intensity Resistance Training with Moderate Blood Flow Restriction Appears Safe and Increases Skeletal Muscle Strength and Size in Cardiovascular Surgery Patients: A Pilot Study. J. Clin. Med. 2021, 10, 547. [CrossRef] [PubMed]

52. Rodrigues, R.; Ferraz, R.B.; Kurimori, C.O.; Guedes, L.K.; Lima, F.R.; de Sá-Pinto, A.L.; Gualano, B.; Roschel, H. Low-load resistance training with blood flow restriction increases muscle function, mass and functionality in women with rheumatoid arthritis. Arthritis Care Res. 2019, 72, 787-797. [CrossRef]

53. Tennent, D.J.; Hylden, C.M.; Johnson, A.E.; Burns, T.C.; Wilken, J.M.; Owens, J.G. Blood flow restriction training after knee arthroscopy: A randomized controlled pilot study. Clin. J. Sport Med. 2017, 27, 245-252. [CrossRef] [PubMed]

54. Campbell, C.M.; Buenaver, L.F.; Finan, P.; Bounds, S.C.; Redding, M.; McCauley, L.; Robinson, M.; Edwards, R.R.; Smith, M.T. Sleep, Pain Catastrophizing and Central Sensitization in Knee Osteoarthritis Patients With and Without Insomnia. Arthritis Care Res. 2015, 67, 1387-1396. [CrossRef] 
55. Hall, M.; Hinman, R.S.; Wrigley, T.V.; Roos, E.M.; Hodges, P.W.; Staples, M.; Bennell, K.L. The effects of neuromuscular exercise on medial knee joint load post-arthroscopic partial medial meniscectomy: "SCOPEX" a randomised control trial protocol. BMC Musculoskelet Disord. 2012, 13, 233. [CrossRef]

56. Crossley, K.W.; Porter, D.A.; Ellsworth, J.; Caldwell, T.; Feland, J.B.; Mitchell, U.; Johnson, A.W.; Egget, D.; Gifford, J.R. Effect of Cuff Pressure on Blood Flow during Blood Flow-restricted Rest and Exercise. Med. Sci. Sports Exerc. 2019, 52, 746-753. [CrossRef]

57. Brandner, C.R.; Warmington, S.A.; Kidgell, D. Corticomotor Excitability is Increased Following an Acute Bout of Blood Flow Restriction Resistance Exercise. Front. Hum. Neurosci. 2015, 9, 652. [CrossRef] [PubMed]

58. Brandner, C.R.; Kidgell, D.; Warmington, S.A. Unilateral bicep curl hemodynamics: Low-pressure continuous vs high-pressure intermittent blood flow restriction. Scand. J. Med. Sci. Sports 2015, 25, 770-777. [CrossRef] [PubMed]

59. Dankel, S.J.; Mattocks, K.T.; Jessee, M.B.; Buckner, S.L.; Mouser, J.G.; Loenneke, J.P. Do metabolites that are produced during resistance exercise enhance muscle hypertrophy? Eur. J. Appl. Physiol. 2017, 117, 2125-2135. [CrossRef] [PubMed]

60. Wilson, J.M.; Lowery, R.P.; Joy, J.M.; Loenneke, J.P.; Naimo, M.A. Practical Blood Flow Restriction Training Increases Acute Determinants of Hypertrophy Without Increasing Indices of Muscle Damage. J. Strength Cond. Res. 2013, 27, 3068-3075. [CrossRef]

61. Salomon, J.A.; Haagsma, J.A.; Davis, A.; de Noordhout, C.M.; Polinder, S.; Havelaar, A.H.; Cassini, A.; Devleesschauwer, B.; Kretzschmar, M.; Speybroeck, N.; et al. Disability weights for the Global Burden of Disease 2013 study. Lancet Glob. Health 2015, 3, e712-e723. [CrossRef]

62. Kakehi, S.; Tamura, Y.; Kubota, A.; Takeno, K.; Kawaguchi, M.; Sakuraba, K.; Kawamori, R.; Watada, H. Effects of blood flow restriction on muscle size and gene expression in muscle during immobilization: A pilot study. Physiol. Rep. 2020, 8, e14516. [CrossRef]

63. Vos, T.; Flaxman, A.D.; Naghavi, M.; Lozano, R.; Michaud, C.; Ezzati, M.; Shibuya, K.; Salomon, J.A.; Abdalla, S.; Aboyans, V.; et al. Years lived with disability (YLDs) for 1160 sequelae of 289 diseases and injuries 1990-2010: A systematic analysis for the Global Burden of Disease Study 2010. Lancet 2012, 380, 2163-2196. [CrossRef]

64. Geneen, L.J.; Moore, R.A.; Clarke, C.; Martin, D.; Colvin, L.A.; Smith, B.H. Physical activity and exercise for chronic pain in adults: An overview of Cochrane Reviews. Cochrane Database Syst. Rev. 2017, 2017, 14651858.

65. Stanford, D.M.; Park, J.; Jones, R.; Credeur, D.P.; McCoy, S.; Jessee, M.B. Acute cardiovascular response to unilateral, bilateral and alternating resistance exercise with blood flow restriction. Eur. J. Appl. Physiol. 2020, 120, 1921-1930. [CrossRef] [PubMed]

66. Neto, G.R.; Sousa, M.S.; Costa, P.B.; Salles, B.F.; Novaes, G.S.; Novaes, J.S. Hypotensive effects of resistance exercises with blood flow restriction. J. Strength Cond. Res. 2015, 29, 1064-1070. [CrossRef] [PubMed] 